MonatsschrKinderheilkd2022·170(Suppl1):S1-S20 https://doi.org/10.1007/s00112-021-01291-w Online publiziert: 6 . September 2021

(c) Springer Medizin Verlag GmbH, ein Teil von Springer Nature 2021

\section{Abstracts 2021: 34th Annual Conference of the Arbeitsgemeinschaft für Pädiatrische Stoffwechselstörungen (APS; Association for pediatric metabolic disorders)}

\section{Tagungsleitung}

Prof. Dr. med. Thomas Opladen (Tagungspräsident)

Prof. Dr. med. René Santer (Vorsitzender der APS)

\section{Grußwort}

Bei der diesjährigen APS-Jahrestagung stehen die angeborenen Störungen der (synaptischen) Neurotransmission im Fokus des wissenschaftlichen Programms. Versierte nationale und internationale Wissenschaftler konnten als Redner gewonnen werden. Sie präsentieren u. a. die Mechanismen der synaptischen Funktion und Dysfunktion im Kontext angeborener Stoffwechselstörungen sowie neue Forschungsergebnisse zu den pathophysiologischen Konzepten und den daraus abgeleiteten (individuellen) Therapiestrategien. Die Erkrankungsgruppe der synaptischen Neurotransmission bildet eine vielseitige Schnittstelle zwischen der Stoffwechselmedizin, der Neuropädiatrie, der Genetik und anderen Disziplinen der Pädiatrie.

In diesem Jahr findet das APS-Stoffwechselseminar vor der Haupttagung statt. Vom 21. bis 22. September 2021 werden dabei Übersichtsthemen zu angeborenen Stoffwechselstörungen und interessante Stoffwechselfälle präsentiert.

Ich freue mich darauf, Sie in Kassel zu begrüßen.

Prof. Dr. med. Thomas Opladen, Heidelberg

Tagungspräsident 


\section{FREE COMMUNICATIONS}

\section{FC01-01 \\ Novel insights expanding the phenotypic spectrum of inherited disorders of biogenic amines}

Kuseyri Hübschmann 0. ${ }^{1}$, Horvath G. ${ }^{2}$, Cortès-Saladelafont E. ${ }^{3}$, Yıldız Y. ${ }^{4}$, Mastrangelo M. ${ }^{5}$, Pons R. ${ }^{6}$, Friedman J. ${ }^{7}$, Mercimek-Andrews S. ${ }^{8}$, Wong S. ${ }^{9}$, Pearson T. ${ }^{10}$, Zafeiriou D. ${ }^{11}$, Kulhánek J. ${ }^{12}$, Kurian M. ${ }^{13}$, López-Laso E. ${ }^{14}$, Oppebøen M. ${ }^{15}$, Jeltsch K. ${ }^{1}$, Garbade S. ${ }^{16}$, Study Group I. ${ }^{17}$, García-Cazorla A. ${ }^{18}$, Opladen T. ${ }^{1}$

'University Children's Hospital Heidelberg, Division of Child Neurology and Metabolic Disorders, Heidelberg, Germany; ${ }^{2}$ University of British Columbia, Department of Pediatrics, Division of Biochemical Genetics, BC Children's Hospital, Vancouver, BC, Canada; ${ }^{3}$ nborn Errors of Metabolism and Child Neurology Unit, Department of Pediatrics, Hospital Germans Trias i Pujol, Badalona and Faculty of Medicine, Universitat Autònoma de Barcelona, Barcelona, Spain; ${ }^{4}$ Hacettepe University, Faculty of Medicine, Department of Pediatrics, Section of Metabolism, Ankara, Turkey; ${ }^{5}$ Department of Human Neuroscience, Unit of Child Neurology and Psychiatry, Università degli Studi di Roma La Sapienza, Rome, Italy ${ }^{6}$ First Department of Pediatrics of the University of Athens, Aghia Sofia Hospital, Athens, Greece; ${ }^{7}$ UCSD Departments of Neuroscience and Pediatrics; Rady Children's Hospital Division of Neurology; Rady Children's Institute for Genomic Medicine; San Diego, USA; ${ }^{8}$ Division of Clinical and Metabolic Genetics, Department of Pediatrics, University of Toronto, The Hospital for Sick Children 555 University Avenue Toronto, ON M5G 1X8, Canada; ${ }^{9}$ Department of Pediatrics and Adolescent Medicine, The Hong Kong Children's Hospital, Hong Kong; ${ }^{10}$ Department of Neurology, Washington University School of Medicine, St. Louis, Missouri, USA; ${ }^{11}$ First Department of Pediatrics Aristotle University of Thessaloniki, Thessaloniki, Greece; ${ }^{12}$ Department of Pediatrics and Inherited Metabolic Disorders, First Faculty of Medicine, Charles University and General University Hospital in Prague, Prague, Czech Republic; ${ }^{13}$ Developmental Neurosciences, UCL Great Ormond Street-Institute of Child Health and Department of Neurology, Great Ormond Street Hospital, London, United Kingdom; ${ }^{14}$ Pediatric Neurology Unit, Department of Pediatrics, University Hospital Reina Sofía, IMIBIC and CIBERER, Córdoba, Spain; ${ }^{15}$ Children's Department Division of Child Neurology Oslo University Hospital Rikshospitalet, Oslo, Norway; ${ }^{16}$ University Children's Hospital Heidelberg, Dietmar-Hopp Metabolic Center, Heidelberg, Germany; ${ }^{17}$ International Working Group on Neurotransmitter related Disorders; ${ }^{18}$ Inborn errors of metabolism Unit, Department of Neurology, Institut de Recerca Sant Joan de Déu and CIBERER-ISCIII, Barcelona, Spain

Introduction: Inherited disorders of neurotransmitter metabolism are rare neurodevelopmental diseases presenting with movement disorders and global developmental delay. They are caused by impaired biosynthesis, breakdown or transport of neurotransmitters, or of their essential cofactors, such as tetrahydrobiopterin.

Patient(s) and Methods: Data of 275 patients (224 new and 51 previously published cases) from 248 families with primary disorders of biogenic amine metabolism from the registry of the International Working Group on Neurotransmitter related Disorders (iNTD) were analyzed in a standardized deep phenotyping approach focusing on clinical and biochemical presentation at disease onset as well as the influence of diagnostic methods.

Results: An increased rate of prematurity was observed in aromatic L-amino acid decarboxylase deficiency (AADCD). Tyrosine hydroxylase (TH) and 6-Pyruvoyl-tetrahydropterin synthase (PTPS) deficiencies presented with high risk for being small for gestational age while patients with PTPS deficiency were also prone to symmetrical intrauterine growth restriction and congenital microcephaly. Age at diagnosis and the diagnostic delay are influenced by diagnostic methods, presence of hyperphenylalaninemia and by disease-specific symptoms. Latency to diagnosis has decreased in recent years, possibly due to novel diagnostic approaches or raised awareness. Although each disorder has a specific biochemical pattern, we observed confounding exceptions to the rule.

Conclusion/Discussion: The study provides comprehensive insights into pre-, peri- and postnatal presentations of inherited disorders of biogenic amines, as well as specific clinical and biochemical patterns in association with diagnostic processes. Our results undermine the importance of careful and systematic clinical evaluation and the value of standardized assessment in longitudinal registries to recognize the potential early signs in evolving phenotypic spectrum and to improve diagnostic approaches.
FC01-02

Intraventricular cerliponase alfa as standard of care in CLN2 disease: 4-year update from an independent ongoing observational study

Schwering C. ${ }^{1}$, Nickel M. ${ }^{1}$, Wibbeler E. ${ }^{1}$, Westermann L. ', Dulz S. ${ }^{2}$, Lezius S. ${ }^{3}$, Maier E. ${ }^{4}$, Schulz A. ${ }^{1}$

'Department of Pediatrics, University Medical Center Hamburg-Eppendorf, Hamburg, Germany; ${ }^{2}$ Department of Ophthalmology, University Medical Center HamburgEppendorf, Hamburg, Germany; ${ }^{3}$ Department of Biometrics, University Medical Center Hamburg-Eppendorf, Hamburg; ${ }^{4}$ Department of Inborn Errors of Metabolism, Dr. von Hauner Children's Hospital, Ludwig Maximilian University, Munich, Germany,

Introduction: Neuronal ceroid-lipofuscinosis type 2 (CLN2) disease, characterised by rapid psychomotor decline, epilepsy and vision loss, is caused by deficiency of the lysosomal enzyme tripeptidyl peptidase 1 (TPP1). Intraventricular enzyme replacement therapy with recombinant human TPP1 (cerliponase alfa) is the only approved treatment. Since 2013, we have treated and followed a total of 59 CLN2 patients both as part of clinical trials as well as standard of care after approval of this treatment at our centre.

Patient(s) and Methods: Out of our cohort of treated CLN2 patients, we evaluated 24 patients ( 14 female, 10 male) who met the following criteria: (i) late infantile phenotype, and (ii) treatment with Cerliponase alfa for at least 6 months as standard of care outside clinical trials settings. Disease progression was monitored by performing the CLN2 clinical rating scale every 3 months. In addition, all patients received 6 monthly follow-up assessments including Denver developmental testing, neurologic exam, seizure inventory, electroencephalogram (EEG), ECG, echocardiography, cerebral MR imaging, and ophthalmologic exams including ocular coherence tomography.

Results: Mean age at symptom onset was 40.1 months (SD 10.6) and mean age at start of enzyme treatment was 61.3 months (SD 27.4). Mean duration of treatment was 24.4 months (SD 14.9) with a maximum of 51 months. Analysis of disease progression was performed similar to ongoing clinical trials using the motor and language domain of the CLN2 clinical rating (ML score) which quantifies the loss of motor and language function on a scale from 6 (normal function) to 0 (no function left). Mean ML score at treatment start was 3.9 (SD 1.6). Treated patients showed an annual decline of 0.52 (SD 0.4) scoring units compared to an annual decline of 1.81 scoring units as previously shown in natural history data from untreated patients.

Conclusion/Discussion: Data from this independent study are based on the largest cohort of patients receiving Cerliponase alfa treatment outside clinical trial settings. Treatment efficacy was analysed not only based on outcome measures used in the original clinical trials but also take into account additional follow-up assessments. 
FC01-03

\section{Cognitive long-term outcome of early diagnosed individuals with glutaric aciduria type 1-a national prospective study over 20 years}

\author{
Maertner E. ${ }^{1}$, Thimm E. ${ }^{2}$, Guder P. ${ }^{3}$, Schiergens K. ${ }^{4}$, Rutsch F. ${ }^{5}$, \\ Roloff S. ${ }^{6}$, Marquardt I. ${ }^{7}$, Das A. ${ }^{8}$, Freisinger P. ${ }^{9}$ Grünert S. ${ }^{10}$, Krämer J. ${ }^{11}$, \\ Baumgartner M. ${ }^{12}$, Beblo S. ${ }^{13}$, Dieckmann A. ${ }^{14}$, Lindner M. ${ }^{15}$, Näke A. ${ }^{16}$, \\ Mühlhausen C. ${ }^{17}$, Maier E. ${ }^{4}$, Kölker S. ${ }^{1}$, Boy N. ${ }^{1}$
}

'Division of Child Neurology and Metabolic Medicine, Centre for Child and Adolescent Medicine, University Hospital Heidelberg, Germany; ${ }^{2}$ Division of Experimental Paediatrics and Metabolism, Department of General Paediatrics, Neonatology and Paediatric Cardiology, University Children's Hospital, Heinrich Heine University Düsseldorf, Düsseldorf, Germany; ${ }^{3}$ Children's Hospital, University Medical Center Hamburg-Eppendorf, Hamburg, Germany; ${ }^{4} \mathrm{Dr}$. von Hauner Children's Hospital, Ludwig-Maximilians-University, Munich, Germany; ${ }^{5}$ Department of General Paediatrics, Metabolic Diseases, University Children's Hospital Muenster, Muenster, Germany; ${ }^{6}$ Charité - Universitätsmedizin Berlin, corporate member of Freie Universität Berlin, Humboldt-Universität zu Berlin, and Berlin Institute of Health, Center for Chronically Sick Children, Berlin, Germany; ${ }^{7}$ Department of Child Neurology, Children's Hospital Oldenburg, Oldenburg, Germany; ${ }^{8}$ Department of Paediatrics, Paediatric Metabolic Medicine, Hannover Medical School, Hannover, Germany; ${ }^{9}$ Children's Hospital Reutlingen, Reutlingen, Germany; ${ }^{10}$ Department of General Paediatrics, Adolescent Medicine and Neonatology, Medical Centre, University of Freiburg, Faculty of Medicine, Freiburg, Germany; ${ }^{11}$ Department of Pediatric Neurology and Inborn Errors of Metabolism, Children's Hospital, University of Ulm, Ulm, Germany; ${ }^{12}$ Division of Metabolism and Children's Research Centre, University Children's Hospital Zurich, Zurich, Switzerland; ${ }^{13}$ Department of Women and Child Health, Hospital for Children and Adolescents, Centre for Paediatric Research Leipzig (CPL), University Hospitals, University of Leipzig, Leipzig, Germany; ${ }^{14}$ Centre for Inborn Metabolic Disorders, Department of Neuropediatrics, Jena University Hospital, Jena, Germany; ${ }^{15}$ Division of Paediatric Neurology, University Children's Hospital Frankfurt, Frankfurt, Germany; ${ }^{16} \mathrm{Children}$ 's Hospital Carl Gustav Carus, Technical University Dresden, Germany; ${ }^{17}$ Department of Pediatrics and Adolescent Medicine, University Medical Center, Göttingen, Germany

Introduction: Glutaric aciduria type 1 (GA1) is a rare inherited neurometabolic disorder of L-lysine metabolism resulting in severe movement disorder with predominant dystonia, if untreated. Implementation into national newborn screening programs with early start of metabolic treatment has dramatically improved neurologic outcome. The two biochemical subtypes, i.e., high and low excreter, are thought to share the same clinical course. Cognitive functions in these individuals are generally thought to be spared of the clinical phenotype but have not systematically been studied yet.

Patient(s) and Methods: This national prospective observational study includes 107 individuals with confirmed GA1 identified by newborn screening between 1999 and 2020 in Germany. Clinical and cognitive (development and IQ) outcome was assessed using standardized tests. Impact of interventional and non-interventional parameters on cognitive outcome was evaluated.

Results: Cognitive tests were available for 72 patients (median age at last test 7.9 years). The majority of younger patients aged eight months up to five years showed normal global development (15 of 24 patients [63\%]) with isolated or global deficits being more frequent in patients with movement disorder (7 of 9 patients [78 \%]). Median IQ of older patients $(n=56$; age range 3.4-19.1 years) was stable with age but significantly lower than in general population (median IQ 87; IQR 78-98). Individuals with high excreter phenotype (median IQ 84; IQR 75-96) showed a significantly poorer performance than individuals with low excreter phenotype (median IQ 98; IQR 92-105; $p=0.0164$ ) whereas sex, clinical motor phenotype and quality of metabolic treatment had no impact on cognitive outcome. Global and subscale IQ results did not differ significantly.

Conclusion/Discussion: Neurologic long-term outcome in individuals with GA1 not only affects motor function but also cognition. Cognitive impairment appears not to be impacted by current therapy and striatal damage. The biochemical high excreter phenotype is the main risk factor for cognitive impairment. These findings implicate the necessity of more effective long-term treatment concepts, especially for high excreting patients.
FC01-04

Lentiviral haematopoietic stem and progenitor cell gene therapy for metachromatic leukodystrophy (MLD): clinical outcomes from 38 patients

Fumagalli F.', Calbi V. ${ }^{2}$, Zambon A. ${ }^{3}$, Gallo V. ${ }^{2}$, Baldoli C. ${ }^{4}$, Rancoita P. ${ }^{5}$, Ciotti F. ${ }^{6}$, Sarzana M. ${ }^{7}$, Segovia J. ${ }^{7}$, Downey G. ${ }^{7}$, Martino S. ${ }^{8}$, Di Serio C. ${ }^{9}$, Ciceri F. ${ }^{10}$, Filippi M. ${ }^{11}$, Bernardo M. ${ }^{2}$, Naldini L. ${ }^{12}$, Sessa M. ${ }^{13}$, Sora M. ${ }^{14}$, Biffi A. ${ }^{15}$, Aiuti A. ${ }^{16}$

'San Raffaele Telethon Institute for Gene Therapy (SR-TIGET), IRCCS San Raffaele Scientific Institute, Milan, Italy; Pediatric Immunohematology Unit and BMT Program, IRCCS San Raffaele Scientific Institute, Milan, Italy; Neurology Department; IRCCS San Raffaele Scientific Institute, Milan, Italy; ${ }^{2}$ San Raffaele Telethon Institute for Gene Therapy (SRTIGET), IRCCS San Raffaele Scientific Institute, Milan, Italy; Pediatric Immunohematology Unit and BMT Program, IRCCS San Raffaele Scientific Institute, Milan, Italy; ${ }^{3}$ Neurology Department, IRCCS San Raffaele Scientific Institute, Milan, Italy; Vita-Salute San Raffaele University, Milan, Italy; Department of Neurology, ASST Papa Giovanni XXIII; Bergamo, Italy; ${ }^{4}$ Neuroradiology Unit, IRCCS San Raffaele Scientific Institute, Milan, Italy; ${ }^{5}$ University Centre of Statistics in the Biomedical Sciences, Vita-Salute San Raffaele University, Milan, Italy; ${ }^{6}$ Pediatric Immunohematology Unit and BMT Program, IRCCS San Raffaele Scientific Institute, Milan, Italy; ${ }^{7}$ Orchard Therapeutics (Europe) Limited; ${ }^{8}$ Department of Chemistry, Biology and Biotechnologies, University of Perugia, Perugia, Italy; ${ }^{9}$ Vita-Salute San Raffaele University, Milan, Italy; ${ }^{10}$ Vita-Salute San Raffaele University, Milan, Italy; Unit of Hematology and Bone Marrow Transplantation, IRCCS San Raffaele Scientific Institute, Milan, Italy; ${ }^{11}$ Neurology Department, IRCCS San Raffaele Scientific Institute, Milan, Italy; Vita-Salute San Raffaele University, Milan, Italy; Neuroimaging Research Unit, Institute of Experimental Neurology (INSPE), Division of Neuroscience, IRCCS San Raffaele Scientific Institute; ${ }^{12}$ San Raffaele Telethon Institute for Gene Therapy (SR-TIGET), IRCCS San Raffaele Scientific Institute, Milan, Italy; Vita-Salute San Raffaele University, Milan, Italy; ${ }^{13}$ San Raffaele Telethon Institute for Gene Therapy (SR-TIGET), IRCCS San Raffaele Scientific Institute, Milan, Italy; Department of Neurology, ASST Papa Giovanni XXIII; Bergamo, Italy; ${ }^{14}$ Neurology Department, IRCCS San Raffaele Scientific Institute, Milan, Italy; ${ }^{15}$ Pediatric Hematology, Oncology and Stem Cell Transplant, Padua University and Hospital, Padua, Italy; Gene Therapy Program, Dana Farber/Boston Children's Cancer and Blood Disorders Center, Boston, MA, USA; ${ }^{16}$ San Raffaele Telethon Institute for Gene Therapy (SR-TIGET), IRCCS San Raffaele Scientific Institute, Milan, Italy; Pediatric Immunohematology Unit and BMT Program, IRCCS San Raffaele Scientific Institute, Milan, Italy; Vita-Salute San Raffaele University, Milan, Italy

Introduction: MLD is a fatal demyelinating lysosomal storage disease resulting from arylsulfatase A (ARSA) deficiency.

Patient(s) and Methods: Thirty-eight patients with early-onset forms of MLD (20 late infantile [LI], 18 early juvenile [EJ]) were treated with haematopoietic stem and progenitor cell (HSPC)-based gene therapy (atidarsagene autotemcel, "arsa-cel"). Arsa-cel consists of autologous $\mathrm{CD}_{3} 4^{+}$cells transduced ex vivo with a lentiviral vector encoding for the functional human ARSA gene. Twenty-nine patients were treated with a fresh formulation of arsa-cel and 9 with a cryopreserved formulation, with follow-up from 1 month to 7.9 years. Following myeloablative busulfan conditioning, the drug product was administered intravenously. Key endpoints were compared to an untreated natural history $(\mathrm{NH})$ cohort.

Results: Of 38 patients treated, 35 are alive ( 2 died from disease progression, 1 from cerebral stroke, all unrelated to treatment with arsa-cel). There were no treatment-related mortality or serious adverse events, no malignancies, no abnormal clonal expansion, and no evidence of replicationcompetent lentivirus. All patients achieved haematological recovery. Stable engraftment of gene-corrected cells and restoration of ARSA activity in the haematopoietic system and cerebrospinal fluid was observed in 33 patients with $\geq 3$ months follow-up. Preliminary data from patients treated with the cryopreserved formulation show engraftment and ARSA activity comparable to those treated with the fresh formulation. The majority of patients treated pre-symptomatically displayed long-term stabilisation of motor function, with many maintaining the ability to walk, and normal cognitive development. Severe motor impairment-free survival was significantly longer in patients treated with the fresh formulation versus the $\mathrm{NH}$ cohort (LI, $\mathrm{p}<0.001 ; \mathrm{EJ}, \mathrm{p}=0.016)$.

Conclusion/Discussion: Data from 38 early-onset MLD patients with up to 7.9 years of follow-up demonstrated that arsa-cel was generally well-tolerated and effective in modifying the disease course of early-onset MLD patients. Atidarsagene autotemcel (tradename: Libmeldy ${ }^{T M}$ ) was approved 
by the European Medicines Agency in 2020 and is indicated for the treatment of children with LI or EJ forms, without clinical manifestations of the disease and in children with the EJ form, with early clinical manifestations of the disease, who still have the ability to walk independently and before the onset of cognitive decline.

\section{FC01-05 \\ Evaluation of the family burden of parents of children with urea cycle defects}

Scharping M. ', Brennenstuhl H. ', Garbade S. ', Posset R. ', Zielonka M. ', Wild B. ${ }^{2}$, Kölker S. ${ }^{1}$, Haun M. ${ }^{2}$, Opladen T. ${ }^{1}$

'Department of Pediatrics, Division for Neuropediatrics and Metabolic Medicine, University of Heidelberg, Germany; ${ }^{2}$ Department of General Internal Medicine and Psychosomatics, Heidelberg University, Heidelberg, Germany,

Introduction: The European registry for Intoxication type Metabolic Diseases ("E-IMD") contains comprehensive information on the clinical and biochemical natural history and outcome of urea cycle disorders (UCDs). However, there is limited information on the burden of affected families and their need for support.

Patient(s) and Methods: To evaluate the family burden of parents with children suffering from UCDs we used validated questionnaires. Besides recording of socio-demographic characteristics, an adapted version of the "Parental Need Scale for Rare Diseases" questionnaire was used. The following categories were examined: (1) Understanding the disease; (2) Working with health professionals; (3) Financial burden; and (4) social, physical, spiritual, and psychological needs. During the telephone interview the parents could give answers on a 5-point Likert scale.

Results: From April to June 202128 female, and 23 male parents from 39 families were interviewed at the University Children's Hospital Heidelberg. OTC was the most frequent disease subtype (female $n=15$, male $n=10$ ) followed by ASS $(n=13)$ and ASL $(n=9)$ deficiency. The mean age of the parents was $47.14(S D=11.75$, range $24-71)$, the mean age of the children 16.46 years $(S D=9.79$, range $2-46$ ). The majority of parents (84$88 \%$ ) reported to feel confident with the understanding of their child's disease. In contrast, $29 \%$ reported a medium or high need for "additional information on the development of their children". $45 \%$ reported a medium or high need for "information on current or future services". Parents (18-29\%) were "dissatisfied" or "extremely dissatisfied" with working with health professionals and their knowledge on IEM. While $25 \%$ reported a financial burden due to paying for special equipment and clothing, $18 \%$ named a financial burden due to paying for childcare. $37 \%$ of the participants indicated a need for support in finding a suitable caretaker and $31 \%$ for additional psychological support.

Conclusion/Discussion: Our study revealed an unmet need for additional psychosocial support for families with children with urea cycle disorders and give suggestion for future family-centered therapeutic concepts. This pilot study can be used as template for the assessment of the family burden in other inherited metabolic diseases.

\section{FC01-06}

The impact of disease severity on the psychological well-being of youth affected by an inborn error of metabolism and their families: A one-year longitudinal study

Dimitrova N.', Glaus J.', Urben S. ', Wüthrich V. ${ }^{1}$, Morisod Harari M.', Ballhausen D. ${ }^{3}$

'Research Unit, Division of Child and Adolescent Psychiatry, Department of Psychiatry, Lausanne University Hospital, Lausanne, Switzerland; ${ }^{2}$ Liaison Psychiatry Unit, Division of Child and Adolescent Psychiatry, Department of Psychiatry, Lausanne University Hospital, Hôpital Nestlé, Lausanne, Switzerland; '3Pediatric unit for metabolic diseases, WomanMother-Child Department, Lausanne University Hospital, Lausanne, Switzerland
Introduction: Inborn errors of metabolism (IEMs) refer to rare heterogeneous genetic disorders with various clinical manifestations that can cause serious physical and psychological sequelae. Results of previous studies on the impact of an IEM on health-related quality of life (HR-QoL) were incongruent and only few studies considered more broadly the psychological well-being of children with IEM and their families. Our objectives were to examine: (1) the impact of the IEM severity on the HR-QoL and psychological functioning of patients and their parents at baseline; and (2) on the evolution over time; and (3) the links and differences between parents' and children's perspectives.

Patient(s) and Methods: The sample included 69 pediatric patients (mean age $=7.55 y, S D=4.59$ ) with evaluations at baseline and after one year. Data on psychological well-being was collected using HR-QoL, Strengths and Difficulties and Cognitive Emotional Regulation Questionnaires. Parental stress and mood difficulties were also measured. IEM severity was rated by a clinician through the INTERMED instrument.

Results: Two groups of children based on IEM severity scores were created ( $n=31$ with low and $n=38$ with moderate/high IEM severity). The two groups differed with respect to age, diet and supplement intake. IEM severity had an impact on HR-QoL and behavioral symptoms in children, as well as on HR-QoL and stress in parents. Moreover, in families with moderate/high IEM severity, the HR-QoL improved after 1-year of follow-up. Conclusion/Discussion: Our findings suggest that children affected by an IEM, particularly with moderate/high severity, and their families benefit, beyond their medical and psychosocial treatment, from the care of an interdisciplinary team including a child psychologist specialized in IEMs, in order to address the impact of the IEM on their physical, cognitive, social and emotional well-being. Future studies should focus on observations over a larger time span, particularly during adolescence, and should include objective measures.

\section{FC02-01 \\ Spectrum and characterization of bi-allelic variants in MMAB causing cblB-type methylmalonic aciduria}

Forny P., Plessl T., Frei C., Bürer C., Froese D., Baumgartner M.

Division of Metabolism and Children's Research Center, University Children's Hospital Zurich, University of Zurich, Zurich, Switzerland

Introduction: Pathogenic variants in $M M A B$ cause $c b / B$-type methylmalonic aciduria, an autosomal-recessive disorder of propionate metabolism. MMAB encodes ATP:cobalamin adenosyltransferase, using ATP and $\mathrm{cob}(\mathrm{I})$ alamin to create $5^{\prime}$-deoxyadenosylcobalamin (AdoCbl), the cofactor of methylmalonyl-CoA mutase (MMUT).

Results: We identified bi-allelic disease-causing variants in $M M A B$ in 97 individuals with $c b / B$-type methylmalonic aciduria, including 33 unique and 16 novel variants. Missense changes accounted for the most frequent pathogenic alleles (p.(Arg186Trp), N=57; p.(Arg191Trp), $N=19$ ); while $c .700 \mathrm{C}>\mathrm{T}$ (p. (Arg234*)) was the most frequently identified truncating variant $(N=14)$. In fibroblasts from 76 affected individuals, the ratio of propionate incorporation in the presence and absence of hydroxocobalamin (PI ratio) was associated to clinical cobalamin responsiveness and later disease onset. We found p.(Arg234*) to be associated with cobalamin responsiveness in vitro, and clinically with later onset; p.(Arg186Trp) and p.(Arg191Trp) showed no clear cobalamin responsiveness and early onset. Mapping these and novel variants onto the MMAB structure revealed their potential to affect ATP and AdoCbl binding. Follow-up biochemical characterization of recombinant MMAB identified its three active sites to be equivalent for ATP binding, determined by fluorescence spectroscopy $\left(K_{d}=21 \mu \mathrm{M}\right)$ and isothermal calorimetry $\left(K_{d}=14 \mu \mathrm{M}\right)$, but function as two non-equivalent AdoCbl binding sites $\left(K_{\mathrm{d} 1}=0.55 \mu \mathrm{M} ; K_{\mathrm{d} 2}=8.4 \mu \mathrm{M}\right)$. Ejection of AdoCbl was activated by ATP $\left(K_{\mathrm{a}}=24 \mu \mathrm{M}\right)$, which was sensitized by the presence of MMUT $\left(K_{\mathrm{a}}=13 \mu \mathrm{M}\right)$.

Conclusion/Discussion: This study expands the landscape of pathogenic $M M A B$ variants, provides association of in vitro and clinical responsive- 
ness, and facilitates insight into MMAB function, enabling better disease understanding.

\section{FC02-02 \\ A spoonful of L-fucose-An efficient therapy for GFUS- CDG, a new glycosylation disorder}

\author{
Feichtinger R. ${ }^{1}$, Hüllen A. ${ }^{2}$, Mayr H. ${ }^{1}$, Thiel C. ${ }^{2}$, Wortmann S. ${ }^{1}$
}

'University Children's Hospital, Salzburger Landeskliniken (SALK) and Paracelsus Medical University (PMU), Salzburg, Austria; ${ }^{2}$ Centre for Child and Adolescent Medicine, Department Pediatrics, University of Heidelberg, Heidelberg, Germany

Introduction: Congenital disorders of glycosylation (CDG) are a genetically and phenotypically heterogeneous family of diseases affecting the coand posttranslational modification of proteins.

Patient(s) and Methods: Using exome sequencing, we detected biallelic variants in GFUS in a patient presenting with global developmental delay, mild coarse facial features and faltering growth.

Results: GFUS encodes GDP-L-fucose synthase, the terminal enzyme in the de novo synthesis of GDP-L-fucose, which is the basis for fucosylation of N- and O-glycans. We found reduced GFUS protein and decreased GDP-L-fucose levels leading to a general hypofucosylation determined in patient's serum glycoproteins, leukocytes, thrombocytes, and fibroblasts. Viral complementation of patient-derived fibroblasts with the wildtype GFUS-cDNA restored normal fucosylation of glycoproteins. Making use of the GDP-L-fucose salvage pathway, oral fucose supplementation was initiated and normalised fucosylation of proteins within four weeks as measured in patient serum and leukocytes. During the follow up of 19 months, a moderate improvement of the growth was seen, as well as a clear improvement of cognitive skills as measured by the Kaufmann $A B C$.

Conclusion/Discussion: In conclusion, GFUS-CDG is a new glycosylation disorder for which oral L-fucose supplementation is promising.

\section{FC02-03 \\ $H P D L$-associated neurological disease-current knowledge and further evidence for a mitochondrial disease-like phenotype}

Husain R. ${ }^{1}$, Grimmel M. ${ }^{2}$, Haack T. ${ }^{2}$, Distelmaier F. ${ }^{3}$, Dierksen N. ${ }^{4}$, Bris C. ${ }^{5}$, Roubertie A. ${ }^{6}$, Dias C. ${ }^{7}$, Singh R. ${ }^{8}$, Siddiqui A. ${ }^{9}$, Brandl U. ${ }^{10}$, Huppke P. ${ }^{10}$, Mentzel H. ${ }^{11}$

'Department of Neuropediatrics, University Hospital Jena, Jena, Germany; ${ }^{2}$ Institute of Medical Genetics and Applied Genomics, University of Tuebingen, Tübingen, Germany; ${ }^{3}$ Department of General Pediatrics, University Children's Hospital, Heinrich-HeineUniversity, Düsseldorf, Germany; ${ }^{4}$ Sozialpädiatrisches Zentrum und Kinderneurologie, Ev. Krankenhaus, Oberhausen, Germany; ${ }^{5}$ Univ Angers, INSERM, CNRS, MITOVASC, Equipe MitoLab, SFR ICAT, Angers, France/Département de Génétique, CHU d'Angers, Angers, France; ${ }^{6} \mathrm{INM}$, University of Montpellier, INSERM, CHU Montpellier, Pediatric Neurology Department, Montpellier, France; ${ }^{7}$ Department of Clinical Genetics, Guy's and St. Thomas' Hospital NHS Foundation Trust, London, United Kingdom/Department of Medical and Molecular Genetics, School of Basic and Medical Biosciences, Faculty of Life Sciences \& Medicine, King's College, Lond; ${ }^{8}$ Children's Neurosciences, Evelina Children's Hospital, Guy's and St Thomas' NHS Foundation Trust, London, United Kingdom; ${ }^{9}$ Department of Neuroradiology, Guy's and St. Thomas' Hospital NHS Foundation Trust, London, United Kingdom; ${ }^{10}$ Department of Neuropediatrics, University Hospital Jena, Jena, Germany; ${ }^{11 S e c t i o n ~ o f ~ P e d i a t r i c ~ R a d i o l o g y, ~ I n s t i t u t e ~ f o r ~ D i a g n o s t i c ~ a n d ~ I n t e r v e n t i o n a l ~ R a d i o l o g y, ~}$ University Hospital Jena, Jena, Germany

Introduction: In 2020 we described a novel pediatric-onset neurodegenerative disease caused by biallelic variants in HPDL. Since then, a total of 80 cases from 51 families have been described. We provide an overview of current knowledge regarding $H P D L$-associated disease and present neuroradiological findings highlighting similarities to Leigh-like syndrome in some patients.

Patient(s) and Methods: HPDL is a single-exon gene encoding 4-hydroxyphenylpyruvate dioxygenase-like protein (HPDL) which was found to be localized to mitochondria. Experimental studies have shown that HPDL is expressed in the nervous system and expression levels increase during neuronal differentiation. It plays a role in motor function in zebrafish. Hpdl knock-out mice show epilepsy, early lethality, smaller brain sizes, and apoptosis of neuronal tissue. Furthermore, HPDL expression was significantly reduced in fibroblasts of affected individuals.

Results: As reported initially, the clinical phenotype is highly variable. All patients developed spasticity predominantly of the lower limbs. The most severe form with neonatal/early infantile-onset disease is characterized by severe neurodevelopmental delay, in some patients complicated by episodes of neurological and respiratory decompensation with elevated blood/CSF lactate. Patients with the mildest phenotype have juvenile-onset with isolated hereditary spastic paraplegia. Further symptoms are microcephaly, epilepsy, demyelinating neuropathy and regression.

51 different disease-associated variants were identified in HPDL (29 missense, 22 loss of-function) either homozygous or compound heterozygous. Most variants were unique within individual families, whereas p.Gly50Asp was present in 8 families. Variants were distributed throughout the gene with most (36/51) affecting a presumed carboxy-terminal catalytic domain.

On brain MRI corpus callosum a-/dysgenesis, cerebral and cerebellar white matter atrophy/signal abnormalities have been described. In some patients fluctuating bilateral midbrain (thalamic, inferior colliculi, inferior olivary nuclei), brain stem, and cerebellar signal changes and even striatal swelling are seen.

Conclusion/Discussion: We present neuroradiological findings in 4 patients (3 unreported) with T2-hyperintense signals bilateral (3) or unilateral (1) in the thalamus and brainstem (1) in part with diffusion restriction (1) matching the initially description. Signal distribution and transient occurrence of these changes that are in part associated with neurological decompensation and elevated lactate places HPDL-associated disease among the mitochondrial disorders, which is in agreement with the localization of the protein.

\section{FC02-04 \\ Effect of ketogenic diet treatment on cellular lipids in murine hippocampal neurons}

\section{Dabke P. ${ }^{1}$, Brogden G. ${ }^{2}$, Naim H. ${ }^{2}$, Das A.}

'Klinik für Pädiatrische Nieren, Leber und Stoffwechselerkrankungen, Medizinische Hochschule Hannover; ' ${ }^{2}$ nstitut für Physiologische Chemie, Tierärztliche Hochschule Hannover

Introduction: Ketogenic diet (KD) is a high fat, low carbohydrate diet used in the treatment of pharmacoresistant epilepsy. Although KD has been in use since a century, its mechanism of action is not completely understood. Neuronal lipids have diverse functions, including maintaining the membrane stability, synapse formation and synaptic transmission. We hypothesized that two KD metabolites-decanoic acid (C10) and beta-hydroxybutyrate (BHB) may alter cellular lipids in murine hippocampal neurons.

Patient(s) and Methods: A hippocampal mouse cell line (HT22) was used as a cell model for this study. Cells were cultured under low $(5.5 \mathrm{mmol} / \mathrm{L})$ glucose concentration in order to simulate a 'ketogenic' environment. Thereafter, cells were grouped as either control (untreated) or KD-treated, wherein either $250 \mu \mathrm{M} \mathrm{C} 10$ or $5 \mathrm{mM} \mathrm{BHB}$ was added to the culture flasks. Cells were cultured for 1 week. Cellular cholesterol was measured using HPLC while phospholipids and sphingomyelin were measured using HPTLC.

Results: Cellular cholesterol levels were lower in both, $\mathrm{C} 10$ and BHB treated HT22 neurons. While the phospholipid content was higher in $\mathrm{C} 10$ incubated cells as compared to the untreated cells, it remained unchanged in BHB incubated cells. However, the ratios of individual phospholipids to cholesterol were higher in cells incubated with both, C10 and BHB.

Conclusion/Discussion: An alteration is the ratios of individual phospholipids to cellular cholesterol is an interesting finding which may point towards a potential alteration in composition and structure of cell and or- 
ganelle membranes. This may be a potential mechanism of therapeutic action of the KD.

\section{FC02-05 \\ Long-term outcomes of individuals with metabolic diseases identified through newborn screening}

Mütze U. ${ }^{1}$, Garbade S. ${ }^{1}$, Gramer G. ${ }^{1}$, Lindner M. ${ }^{2}$, Freisinger P. ${ }^{3}$, Grünert S. ${ }^{4}$, Hennermann J. ${ }^{5}$, Ensenauer R. ${ }^{6}$, Thimm E. ${ }^{7}$, Zirnbauer J. ${ }^{9}$, Leichsenring M. ${ }^{9}$, Gleich F.', Hörster F.', Grohmann-Held K. ${ }^{10}$, Boy N. ', Fang-Hoffmann J.', Burgard P.' , Walter M. ', Hoffmann G. ${ }^{\prime}$, Kölker S. '

'Division of Child Neurology and Metabolic Medicine, Center for Child and Adolescent Medicine and Dietmar Hopp Metabolic Center, University Hospital Heidelberg, Heidelberg, Germany; ${ }^{2}$ Division of Pediatric Neurology, University Children's Hospital Frankfurt, Frankfurt, Germany; ${ }^{3}$ Children's Hospital Reutlingen, Klinikum am Steinenberg, Reutlingen, Germany; ${ }^{4}$ Department of General Pediatrics, Adolescent Medicine and Neonatology, Medical Center - University of Freiburg, Faculty of Medicine, Freiburg, Germany; ${ }^{5}$ Villa Metabolica, Dept. of Pediatric and Adolescent Medicine, University Medical Center Mainz, Mainz, Germany; ${ }^{6}$ Department of General Pediatrics, Neonatology, and Pediatric Cardiology, University Children's Hospital, Heinrich Heine University Düsseldorf, Düsseldorf, Germany; Institute of Child Nutrition, Max Rubner-Institut, Karlsruhe, Germany; ${ }^{7}$ Department of General Pediatrics, Neonatology, and Pediatric Cardiology, University Children's Hospital, Heinrich Heine University Düsseldorf, Düsseldorf, Germany; ' $U l m$ Department of Pediatric and Adolescent Medicine, Ulm University Medical School, Ulm, Germany; ${ }^{10} \mathrm{Center}$ for Child and Adolescent Medicine, University Hospital Greifswald, Greifswald, Germany

Introduction: Although extended newborn screening (NBS) programs have been introduced more than 20 years ago, their impact on the longterm clinical outcome of individuals with inherited metabolic diseases (IMDs) is still rarely investigated.

Patient(s) and Methods: We studied the clinical outcomes of individuals with IMDs identified by NBS between 1999 and 2016 in a prospective multi-center observational study.

Results: In total, 306 screened individuals with IMDs, 115 with phenylketonuria (PKU) and 191 with other IMDs with a life-long risk for acute metabolic decompensation, were followed for a median time of 6.2 years. Although the risk for metabolic decompensation was disease-specific and NBS could not prevent decompensations in every individual at risk $(N=49)$, the majority did not develop permanent disease-specific signs (75.9\%), showed normal development $(95.6 \%)$ and normal cognitive outcome (87.7\%, mean IQ 100.4), and mostly attended regular kindergarten $(95.2 \%)$ and primary school (95.2\%). This demonstrates that not only individuals with PKU, serving as a benchmark, but also those with life-long risk for metabolic decompensation had a favourable long-term outcome. High NBS process quality is the prerequisite of this favourable outcome. This is supported by 28 individuals presenting with first symptoms at median age (IQR; range) of 3.5 days $(0-5 ; 0-9)$ before NBS results were available, the absence of neonatal decompensations after the report of NBS results, and by the challenge of keeping relevant process parameters at a constantly high level.

Conclusion/Discussion: Conclusions: NBS for IMDs, although not completely preventing clinical presentations in all individuals, can be considered a highly successful program of secondary prevention.

\section{FC02-06 \\ Newborn screening and disease variants predict neurological outcome in isovaleric aciduria}

Henze L. ${ }^{1}$, Mütze U. ${ }^{1}$, Gleich F., Lindner M. ${ }^{2}$, Grünert S. ${ }^{3}$, Spiekerkoetter U. ${ }^{3}$, Santer R. ${ }^{4}$, Blessing H. ${ }^{5}$, Thimm E. ${ }^{6}$, Ensenauer R. ${ }^{7}$, Weigel J. , Beblo S. ${ }^{9}$, Arélin M. ${ }^{9}$, Hennermann J. ${ }^{10}$, Marquardt T. ${ }^{11}$, Marquardt I. ${ }^{12}$, Freisinger P. ${ }^{13}$, Krämer J. ${ }^{14}$, Dieckmann A. ${ }^{15}$, Weinhold N. ${ }^{16}$, Keller M. ${ }^{1}$, Walter M. ${ }^{1}$, Schiergens K. ${ }^{17}$, Maier E. ${ }^{17}$, Hoffmann G. ${ }^{1}$, Garbade S. ${ }^{1}$, Kölker S. ${ }^{1}$

'Division of Child Neurology and Metabolic Medicine, Center for Child and Adolescent Medicine and Dietmar Hopp Metabolic Center, University Hospital Heidelberg, Heidelberg, Germany; ${ }^{2}$ Division of Pediatric Neurology, University Children's Hospital Frankfurt, Frankfurt, Germany; ${ }^{3}$ Department of General Pediatrics, Adolescent Medicine and Neonatology, Medical Center - University of Freiburg, Faculty of Medicine, Freiburg, Germany; ${ }^{4}$ Dept of Pediatrics, University Medical Centre Eppendorf, Hamburg, Germany; ${ }^{5}$ Kinder- und Jugendklinik, Universitätsklinikum Erlangen, Erlangen, Germany; ${ }^{6}$ Department of General Pediatrics, Neonatology, and Pediatric Cardiology, University Children's Hospital, Heinrich Heine University Düsseldorf, Düsseldorf, Germany; ${ }^{7}$ Department of General Pediatrics, Neonatology, and Pediatric Cardiology, University Children's Hospital, Heinrich Heine University Düsseldorf, Düsseldorf, Germany; Institute of Child Nutrition, Max-Rubner-Institut, Karlsruhe, Germany; ${ }^{8}$ Praxis für Kinder- und Jugendmedizin, Endokrinologie und Stoffwechsel, Augsburg, Germany; ${ }^{\circ}$ Department of Women and Child Health, Hospital for Children and Adolescents, Center for Pediatric Research Leipzig (CPL), University Hospitals, University of Leipzig, Leipzig, Germany; ${ }^{10} \mathrm{Villa}$ Metabolica, Dept. of Pediatric and Adolescent Medicine, Mainz University Medical Center, Mainz, Germany; "Department of General Pediatrics, Metabolic Diseases, University Children's Hospital Muenster, Muenster, Germany; ${ }^{12}$ Department of Child Neurology, Children's Hospital Oldenburg, Oldenburg, Germany; ${ }^{13}$ Children's Hospital Reutlingen, Klinikum am Steinenberg, Reutlingen, Germany; ${ }^{14}$ University of Ulm, Department of Pediatric and Adolescent Medicine, Ulm, Germany; ${ }^{15}$ Center for Inborn Metabolic Disorders, Department of Neuropediatrics, Jena University Hospital, Jena, Germany; ${ }^{16} \mathrm{C}$ harité - Universitätsmedizin Berlin, corporate member of Freie Universität Berlin, Humboldt-Universität zu Berlin, and Berlin Institute of Health, Center for Chronically Sick Children, Berlin, Germany; ${ }^{17}$ Dr. von Hauner Children's Hospital, Ludwig-Maximilians-University, Munich, Germany

Introduction: Isovaleric aciduria (IVA), an organic aciduria with severe (classic IVA) or attenuated phenotype (mild IVA), is included in newborn screening (NBS) programs worldwide. The long-term clinical benefit of screened individuals, however, is still rarely investigated.

Patient(s) and Methods: National, prospective, observational, multi-centre study of individuals with confirmed IVA identified by NBS between 1998 and 2018. Comparison to previously published cohorts of symptomatically diagnosed patients.

Results: Long-term clinical outcomes of 94 individuals with IVA were evaluated, representing $73.4 \%$ (for classic IVA: $92.3 \%$ ) of the German NBS cohort. In classic IVA $(N=24)$, NBS prevented untimely death except in one individual with lethal neonatal sepsis (3.8\%) showing the benefit of NBS compared to pre-screening-era $(18.7-43.2 \%, P=0.08, P=0.0078$, respectively). However, NBS did not completely prevent single $(N=10)$ or recurrent $(N=7)$ metabolic decompensations, 13 of them occurring already neonatally. IQ (mean \pm SD, $90.7 \pm 10.1$ ) was mostly normal but below the reference population $(P=0.0022)$ and was even lower in individuals with severe neonatal decompensations (IQ 78.8 \pm 7.1 ) compared to those without crises (IQ 94.7 $\pm 7.5^{\circ} P=0.01$ ). Similar results were obtained for school placement. In contrast, individuals with mild IVA had excellent neurocognitive outcomes (IQ 105.5 \pm 15.8 ; normal school placement) and a benign disease course (no metabolic decompensation, normal hospitalisation rate), which did not appear to be impacted by metabolic maintenance therapy.

Conclusion/Discussion: NBS reduces mortality in classic IVA, but does not reliably protect against severe neonatal metabolic decompensations, crucial for favourable neurocognitive outcome. In contrast, individuals with mild IVA had excellent clinical outcomes regardless of metabolic maintenance therapy, questioning their benefit from NBS. Harmonized stratified therapeutic concepts are urgently needed. 
FC02-07

Congenital lactase deficiency: Molecular and cellular analysis of intestinal lactase-phlorizin hydrolase gene variants

\begin{abstract}
Marten L. ${ }^{1}$, Wanes D. ${ }^{2}$, Stellbrink T. ${ }^{2}$, Santer R. ${ }^{3}$, Naim H. ${ }^{2}$
${ }^{1}$ Department of Biochemistry, University of Veterinary Medicine Hannover, Hannover, Germany; 2. Department of Pediatrics, University Medical Center Eppendorf, Hamburg, Germany; ${ }^{2}$ Department of Biochemistry, University of Veterinary Medicine Hannover, Hannover, Germany; ${ }^{3}$ Department of Pediatrics, University Medical Center Eppendorf, Hamburg, Germany
\end{abstract}

Introduction: Background: Congenital lactase deficiency (CLD) is a rare autosomal recessive genetic disorder caused by mutations in the LCT gene leading to a dysfunctional lactase-phlorizin hydrolase (LPH). LPH is a ß-galactosidase located in the intestinal brush border membrane and indispensable for lactose digestion. Impaired LPH-function results in severe symptoms in the neonatal period, including watery diarrhea and dehydration, leading to potential life-threatening disease of the newborn. While several hypomorphic variants of the LCT gene have been characterized, their implications on the enzymatic activities, protein trafficking and cellular localization of LPH still need to be elucidated.

Patient(s) and Methods: Methods: This study aims to establish the genotype-phenotype correlation of LPH mutations p.Y1390X, p.E1612X, p.S1150Pfs*19, p.S1121L, p.R1587H, and p.S688P that have been identified in patients from different ethnic groups in homozygous or compound heterozygous patterns of inheritance. For this purpose, LPH mutants were generated from wild type LPH by site-directed mutagenesis transiently expressed in COS-1 cells and their structural and functional features analyzed.

Results: Results: The results demonstrated phenotypic variations in the function, structure, and intracellular protein trafficking of the mutants in comparison to wild type LPH. Thus, the LPH mutants are either entirely enzymatically inactive or reveal a substantial reduction in their digestive capacity towards lactose. These LPH mutants are transport incompetent and localized to the ER as determined by (i) the sensitivity of their biosynthetic forms to endoglycosidase $\mathrm{H}$, which cleaves immature mannose-rich glycosylated ER-located precursor forms of glycoproteins and (ii) their colocalization with an ER marker in immunofluorescence images.

Conclusion/Discussion: Discussion: Our findings expand the current knowledge on the biochemical properties and function of LPH variants as well as the mechanisms involved in the pathogenesis of CLD and their relationship to other carbohydrate malabsorption disorders. The knowledge gained from this study is essential in determining the potential effects of LPH mutants on their wild type counterpart in heterozygote carriers.

\section{FC02-08 \\ Endothelial colony forming cells as a primary cell model of Fabry disease}

\section{Dohr K. ', Gastager-Ehgartner M. ', Cvitic S. ${ }^{1}$}

'Medical University of Graz/Department of Paediatrics and Adolescent Medicine/Research Unit of Analytical Mass Spectrometry, Cell Biology and Biochemistry of Inborn Errors of Metabolism,

Introduction: Fabry disease (FD) is a rare lysosomal storage disorder caused by a deficiency of the enzyme alpha-galactosidase $\mathrm{A}$, resulting from mutations in the GLA gene. It is characterized by accumulation of glycolipids, mainly globotriaosylceramide (Gb3) within the lysosomes of many cell types including endothelial cells, and secretion of globotriaosylsphingosine (lyso-Gb3) into body fluids. As a multisystemic disorder, FD is characterized by a dysfunctional endothelium with cardiovascular complications as the leading cause of mortality. We aimed to establish a representative primary cell model of FD which would in contrast to the currently used HEK model enanble genotype-phenotype correlation at the cellular level beyond enzyme activity. Endothelial colony forming cells (ECFCs) are circulating progenitors that sustain endothelial repair, angiogenesis and vascular growth. ECFCs are easily isolated from peripheral blood and can be cultured for several passages in vitro.

Patient(s) and Methods: We isolated endothelial progenitor cells from peripheral blood from patients suffering from FD as well as from healthy control donors and differentiated them to ECFCs. Quality control was performed by immunocytochemistry using antibodies against endothelial cell markers (CD31, von Willebrand factor (VWF)), fibroblast markers (CD90, TE-7) and muscle cell markers (smooth muscle actin (SMA), Desmin). Substrate accumulation in lysosomes was determined by immunofluorescence co-staining of Gb3 and a lysosomal marker LAMP2a. Concentration of lysoGb3 in culture supernatant was determined by LC-MS/MS.

Results: ECFCs isolated from Fabry patients took longer to build colonies and to reach confluence indicating potential differences in differentiation and proliferation when compared to ECFCs from healthy patients. Immunofluorescence co-staining of Gb3 and LAMP2a showed increased deposition of Gb3 in lysosomes in parallel with increased lyso-Gb3 concentration in culture supernatants of ECFCs from Fabry vs healthy donors.

Conclusion/Discussion: ECFCs isolated from patients with FD display main characteristics of the pathology, namely intracellular and extracellular substrate accumulation that may affect cellular function such as cell growth and consequently cause endothelial dysfunction. Further characterization of ECFCs from Fabry patients eg gene transcription, DNA methylation and functional analysis is planned to gain insights into the endothelial pathophysiology of FD and to test the applicability of this cell model for drug repurposing studies and precision medicine.

\section{FC02-09 \\ Impact of Fabry disease on mitochondrial homeostasis in renal epithelial cells}

Schumann A. ', Schaller K.', Belche V., Cybulla M. ${ }^{2}$, Grünert S. ', Moers N. ${ }^{3}$, Sass J. ${ }^{3}$, Kaech A. ${ }^{4}$, Hannibal L.,, Spiekerkötter U.'

'Department of General Paediatrics, Adolescent Medicine and Neonatology, Medical CenterUniversity of Freiburg, Faculty of Medicine, Freiburg, Germany; ${ }^{2}$ Center of Internal Medicine, Department of Nephrology and Rheumatology, Fachinternistische Gemeinschaftspraxis Markgraeflerland, Muellheim, Germany; ${ }^{3}$ Institute for Functional Gene Analytics (IFGA) \& Department of Natural Sciences, Bonn-Rhein-Sieg University of Applied Sciences, Rheinbach, Germany; ${ }^{4}$ Center for Microscopy and Image Analysis, University of Zurich, Zurich, Switzerland; ${ }^{5}$ Department of General Paediatrics, Adolescent Medicine and Neonatology, Laboratory of Clinical Biochemistry and Metabolism, Medical Center-University of Freiburg, Faculty of Medicine, Freiburg, Germany

Introduction: Fabry disease (FD) is caused by deficiency of the lysosomal enzyme alpha-galactosidase following $X$-linked inheritance. Storage of glycosphingolipids and Gb3 in particular leads to multi organ involvement. Cardiovascular complications and progressive renal failure are severe, life-threatening long-term complications. Enzyme replacement therapy fails to sufficiently prevent chronic kidney disease (CKD) progression. The key players driving CKD in FD and the involvement of renal tubular cells for disease progression remain to be elucidated.

Methods: Human renal tubular cell lines isolated from the urine of healthy male controls and FD patients were investigated by electron microscopy, immuno-fluorescent staining, immuno-blotting, and liquid chromatography-tandem mass spectrometry.

Results: To perform energy consuming transport and secretion processes, renal tubular cells are lined by numerous mitochondria. Our studies revealed major changes of mitochondrial morphology and function in FD cells. These changes were accompanied by alterations in the expression of TCA-cycle intermediates and differences in substrate utilization for energy production, e. g., enhanced consumption of fatty acids in FD cells. We observed activation of autophagy in FD cells which seems to impact on mitochondrial homeostasis and energy metabolism. Sirtuin1, a $\mathrm{NAD}^{+}$-dependent deacetylase exerting anti-inflammatory and anti-fibrotic effects, was up-regulated in FD cells. Principal component analysis re- 
vealed distinct metabolic clustering of FD cells as compared to healthy controls and other renal disease models investigated.

Conclusion/Discussion: Our data revealed a relevant impact of lysosomal FD on renal mitochondrial homeostasis and energy metabolism. A better understanding of underlying pathomechanisms on a tissue-specific level may identify new, potentially targetable aims and improve treatment performance. The role of impaired energy metabolism in FD is subject of further investigation ${ }^{1}$.

\section{POSTERS:}

\section{P01}

\section{Never too old for an inborn metabolic disorder}

Arélin M. ${ }^{1}$, Rohde C. ${ }^{1}$, Kirmse S. ' , Metelmann M. ${ }^{2}$, Ceglarek U. ${ }^{3}$, Then Bergh F. ${ }^{2}$, Beblo S. ${ }^{1}$

${ }^{1}$ Hospital for Children and Adolescents, Center for Pediatric Research Leipzig (CPL), Department of Women and Child Health, University Hospitals, University of Leipzig, Germany; ${ }^{2}$ Department of Neurology, University Hospitals, University of Leipzig, Germany; ${ }^{3}$ Institute of Laboratory Medicine, Clinical Chemistry and Molecular Diagnostics, University Hospital Leipzig, Germany

Introduction: We report a 28 year old lady who presented with severe ketoacidosis ( $\mathrm{pH} 7.15)$, hypoglycemia $(<2 \mathrm{mmol} / \mathrm{l})$ and elevated liver enzymes at $26+5$ weeks gestation. She complained of muscular weakness and dyspnea since the 16th week of gestation. HELLP-syndrome was assumed and the child was delivered by C-section and died within the first two days of life. The mother slowly recovered but muscular weakness persisted. Over the next year she developed flaccid proximal tetraparesis without electromyographic abnormality. Muscle biopsy showed MHC expression but no lymphocytic infiltrates; fibres contained fat depositions. Assuming polymyositis she received corticosteroids and slowly improved. She subsequently developed dysphagia, was admitted to the neurologically ICU and treated with high dose steroids and azathioprine, although dissociative disorder was discussed. When the course remained unstable escalation to cyclophosphamide was scheduled, thorough review of past results raised doubts about inflammatory disorder and prompted metabolic workup. This revealed typical acylcarnitine profile in dried blood as well as elevated concentrations of glutaric acid, adipic acid and ethylmalonic acid compatible with multiple acyl CoA dehydrogenase deficiency. Under immediate treatment with riboflavin and carbohydrate rich meals, the patient improved over weeks to complete remission and has since given birth to two healthy children after unremarkable pregnancies.

\section{P02}

\section{Challenges facing patients in PKU therapy and experience in support patients}

von Hopffgarten A. ${ }^{1}$, Kraft T. ${ }^{1}$

'IQVIA CSMS GmbH, Mannheim, Germany

Introduction: Phenylketonuria (PKU) a rare, congenital metabolic disorder in which phenylalanine (Phe) is not or insufficiently converted into tyrosine, thus accumulating in the brain and impairing brain development and function. Among other things, it leads to significant cognitive impairments as well as behavioral and movement disorders. The goal of PKU treatment is to lower Phe levels in the blood through a low-protein diet, treatment with $\mathrm{BH} 4$ or enzyme replacement therapy. Phe control is necessary from birth and throughout life. Adherence to treatment regimens is challenging for a broad cross-section of patients with chronic disease.
Patient(s) and Methods: The program is aimed for PKU patients and their carers. The majority of 173 patients show difficulty in performing everyday tasks. Some require motivational support due to transition points in their lives, pregnancy, with therapy initiation, dealing with side effects or adjusting their diet from low protein to natural.

Review of available literature regarding challenges facing PKU patients, evaluation, analysis of patient interviews and data from the ongoing program, outlining framework of support based on the results of the analysis and interviews

Results: Especially during the COVID-19 pandemic, there has been an increase in digital channels, but $\mathrm{f} 2 \mathrm{f}$ support remains invaluable in building a trusting relationship with patients. This diversity is due in part to the complexity of the content to be communicated.

Patients and treatment centers see an intensive need for complex issues and therapies to ensure a sustainable success. A good relationship of trust between patient and nurse provides insight into motivators, barriers and the home environment; a well-coordinated cooperation between $\mathrm{HCP}$, patient and nurse prepares the right path to the therapy goal.

Conclusion/Discussion: The analysis of patient related challenges and of the various guidelines and position papers available reinforce the need for structured and broad support for PKU patients in order to ensure optimal disease management. The experience gathered from the patient support program underlines the need for integrated supervision and support of patients working closely with the treatment centers.

\section{P03}

Improved motor function in children with AADC deficiency treated with eladocagene exuparvovec (PTCAADC): interim findings from a phase 2 trial

Hwu P. ${ }^{1}$, Chien Y. ${ }^{1}$, Lee N. ${ }^{1}$, Tseng S. ${ }^{1}$, Conway A. ${ }^{2}$, Pykett M. ${ }^{2}$, Tai C. ${ }^{1}$

'National Taiwan University Hospital, Taipei, Taiwan; ${ }^{2}$ PTC Therapeutics, Lynnfield, Massachusetts, USA,

Introduction: Aromatic L-amino acid decarboxylase (AADC) deficiency is a rare genetic disorder caused by mutations in $D D C$, resulting in missed motor milestones. We report interim findings from a phase 2 study on gene therapy with PTC-AADC.

Patient(s) and Methods: Eight children underwent bilateral intraputaminal injection of PTC-AADC $\left(1.8 \times 10^{11} \mathrm{vg}\right.$ for $\geq 3$ years and $02.4 \times 10^{11} \mathrm{vg}$ for $<3$ years of age). Endpoints are described in table 1. Mean follow-up was 11.5 months.

Results: Patients received doses of $1.8 \times 10^{11} \mathrm{vg}(n=3$; mean age, 55.0 months) or $2.4 \times 10^{11} \mathrm{vg}(n=5$; mean age, 24.8 months). Baseline PDMS-2 and AIMS total scores were low. At 1 year, some patients had achieved motor milestones (table 2). Increases from baseline in PDMS-2, AIMS, and Bayley-III total scores at 1 year were statistically significant $(P<0.0001$, $P \leq 0.0016$, and $P \leq 0.0004$ respectively, both doses). Mean body weight increased from baseline to year 1 in both groups. The number of patients with hypotonia, oculogyric crises, and limb dystonia decreased during the first year. No viral shedding was detected. No differences were observed in the safety profiles for the doses. All patients experienced $\geq 1 \mathrm{TEAE}$, most of mild intensity; none were definitely related to treatment. Mild dyskinesia episodes were considered possibly related to therapy. Of 21 serious AEs, all resolved and were considered unlikely to be related to study treatment. Conclusion/Discussion: Children with AADC deficiency achieved meaningful gains in motor function 1 year after PTC-AADC. No new safety signals were observed. 


\section{P04}

Improved motor function in children with AADC (AADC) deficiency treated with eladocagene exuparvovec (PTCAADC): interim findings from a phase $1 / 2$ study

Hwu P.', Chien Y.', Lee N. ', Tseng S. ', Conway A. ${ }^{2}$, Pykett M. ${ }^{2}$, Tai C. ${ }^{1}$

${ }^{1}$ National Taiwan University Hospital, Taipei, Taiwan; ${ }^{2}$ PTC Therapeutics, Lynnfield, Massachusetts, USA

Introduction: AADC deficiency is caused by mutations in the gene encoding the enzyme AADC, resulting in dopamine deficiency and movement disorders. Current treatments fail to restore dopamine levels and improve motor function. We report interim findings from a study evaluating efficacy and safety of PTC-AADC, a recombinant adeno-associated virus vector containing human CDNA encoding AADC.

Patient(s) and Methods: This is a 2-year interim analysis from a 5-year, phase $1 / 2$, prospective, open-label trial in AADC deficiency using bilateral intraputaminal injections of $1.8 \times 10^{11} \mathrm{vg}$ PTC-AADC. Endpoints and assessments are shown (table). Mean follow-up was 39.9 months.

Results: Of 10 patients (median age 34.0 months) treated, 1 withdrew at 11 months (influenza B encephalopathy leading to death). All others completed follow-up through year 2. Motor function scales significantly improved over 4 years (figure). Mean body weight increased in year 1 $(P=0.0011)$. Hypotonia, oculogyric crises, limb dystonia, and stimulus provoked dystonia decreased. Mean putaminal ${ }^{18} \mathrm{~F}$-DOPA uptake increased by year 1 and through year 2 . No viral shedding was detected. All patients experienced $\geq 1$ TEAE (mostly mild or moderate); none were considered definitely treatment-related. 27/131 TEAEs were possibly treatment-related, including 18 dyskinesia episodes; most resolved within 6 months. Seven patients experienced 18 serious AEs; all resolved except influenza $B$ encephalopathy in 1 patient resulting in death. All were considered unrelated to treatment.

Conclusion/Discussion: Children with AADC deficiency achieved improvements in motor function after intraputaminal PTC-AADC therapy. No new safety signals were identified. These findings support the efficacy and safety of PTC-AADC in AADC deficiency.

\section{P05}

Improved motor function in children with AADC deficiency treated with eladocagene exuparvovec (PTCAADC): compassionate use study

\author{
Hwu P. ${ }^{1}$, Chien Y. ${ }^{1}$, Lee N. ${ }^{1}$, Tseng S. ${ }^{1}$, Conway A. ${ }^{2}$, Pykett M. ${ }^{2}$, Tai C. ${ }^{1}$ \\ ${ }^{1}$ National Taiwan University Hospital, Taipei, Taiwan; ${ }^{2}$ PTC Therapeutics, Lynnfield, \\ Massachusetts, USA
}

Introduction: AADC deficiency is caused by mutations in the gene for AADC, movement disorders. This study evaluated efficacy and safety of PTC-AADC, a recombinant adeno-associated virus containing CDNA encoding AADC, in children with AADC deficiency for 5 years.

Patient(s) and Methods: This study was a single-arm, compassionate-use trial enrolling children $>2$ years of age with AADC deficiency $(n=8)$ who received intraputaminal PTC-AADC. Primary efficacy endpoint was the proportion achieving key milestones using the Peabody Developmental Motor Scale, Second Edition (PDMS-2), compared with a historical control group $(n=82)$. Secondary endpoints included changes in PDMS-2, Alberta Infant Motor Scale (AIMS), and Comprehensive Developmental Inventory for Infants and Toddlers (CDIIT) scores and body weight. Pharmacodynamic endpoints included putaminal ${ }^{18} \mathrm{~F}$-DOPA uptake on positron emission tomography (PET). Safety endpoints included treatment-emergent adverse events (TEAEs) and viral shedding. Mean follow-up duration was 62.5 months.

Results: PTC-AADC increased motor milestone achievement (table). Mean PDMS-2, AIMS, and CDIIT total scores (all $P<0.0001$ ) and mean body weight $(P=0.027)$ increased from baseline to 5 years. Putaminal ${ }^{18} \mathrm{~F}$-DOPA PET uptake increased over time $(P=0.0134)$ (figure). All patients experienced $\geq 1$ TEAE none considered PTC-AADC related and most of mild/ moderate intensity. Eight patients experienced 9 possibly/probably treatment-related dyskinesia episodes, generally in the first few months and resolving within 4 months. Seven patients experienced $\geq 1$ serious $A E$, none PTC-AADC related. No deaths occurred during the study. No viral shedding was detected.

Conclusion/Discussion: Children with AADC deficiency achieved sustained improvements in motor function after PTC-AADC, with increased putaminal dopamine production. No new safety signals were identified.

P06

Reductions in oculogyric crisis duration and frequency in children with aromatic L-amino acid decarboxylase deficiency treated with eladocagene exuparvovec gene therapy: results from 3 clinical trials

Hwu P. ${ }^{1}$, Chien Y. ${ }^{1}$, Lee N. ${ }^{1}$, Tseng S. ${ }^{1}$, Wang A. ${ }^{2}$, Schilling T. ${ }^{2}$, Wang J. ${ }^{2}$, Kristensen A. ${ }^{2}$, Ozdas S. ${ }^{3}$, Tai C. ${ }^{1}$

'National Taiwan University Hospital, Taipei, Taiwan; ${ }^{2}$ PTC Therapeutics, Inc., South Plainfield, New Jersey, USA; ${ }^{3}$ PTC Therapeutics Switzerland GmbH, Zug, Switzerland

Introduction: Aromatic l-Amino Acid Decarboxylase (AADC) deficiency is a rare autosomal recessive disorder resulting in marked dopamine loss, impeding normal motor development. A common symptom of AADC deficiency is oculogyric crises (OGC), which is frequently linked to decreased dopamine levels and characteristic involuntary eye movement. OGCs can also be accompanied by limb stiffness, torso rigidity, and autonomic signs. Eladocagene exuparvovec, a recombinant adeno-associated viral vector containing the human CDNA encoding the AADC enzyme, was studied in 3 AADC clinical trials.

Patient(s) and Methods: Eladocagene exuparvovec was administered as a bilateral infusion in the putamen of 28 children with AADC deficiency in 3 clinical trials (AADC-CU/1601 [8 patients, completed], AADC-010 [10 patients, ongoing], and AADC-011 [10 patients to date; ongoing]). Patients received a total of $1.8 \times 10^{11} \mathrm{vg}(n=21)$ or $2.4 \times 10^{11} \mathrm{vg}(n=7$; AADC-011)]. Duration ( $h / w k$ ) and frequency (episodes/wk) of OGC episodes were calculated at baseline and 3 to 12 months after gene therapy.

Results: Burden of OGC episodes decreased steadily following treatment with eladocagene exuparvovec. At baseline, mean duration of OGC episodes was $12.58 \mathrm{~h} / \mathrm{wk}(n=22)$. OGC was reduced from baseline by a mean of $2.08 \mathrm{~h} /$ wk at 3 months ( $n=20), 2.24 \mathrm{~h} /$ wk at 6 months $(n=12), 3.2 \mathrm{~h} / \mathrm{wk}$ at 9 months $(n=12)$, and $3.64 \mathrm{~h} /$ wk at 12 months $(n=8)$. At baseline, mean frequency of OGC was 2.63 episodes/wk $(n=22)$. By month 3 , the mean frequency decreased to 1.93 episodes/wk $(n=20)$, by month 6 , it was 1.9 episodes/wk $(n=12)$, and remained at $\sim 2$ episodes/wk from months 9 to 12.

Conclusion/Discussion: These results indicate a pattern of steady and sustained decrease over time in OGC episodes after PTC-AADC gene therapy. Reduced OGC has the potential to improve quality of life for patients with AADC deficiency and their caregivers by decreasing clinical burden. 
P07

\section{CDG-Ilb syndrome: In-depth glycomic characterization of a novel patient using xCGE-LIF}

Beimdiek J. ${ }^{1}$, Hennig R. ${ }^{2}$, Burock R. ${ }^{2}$, Puk O. ${ }^{3}$, Biskup S. ${ }^{3}$, Rapp E. ${ }^{2}$, Lesinski-Schiedat A. ${ }^{4}$, Buettner F. ${ }^{1}$, Das A. ${ }^{5}$

${ }^{1}$ Institute of Clinical Biochemistry, Hannover Medical School; ${ }^{2}$ glyXera GmbH, Magdeburg; ${ }^{3}$ Praxis für Humangenetik, Tübingen; ${ }^{4}$ Otorhinolaryngology, Hannover Medical School;

${ }^{5}$ Clinic for Pediatric Kidney-, Liver-, and Metabolic Diseases, Hannover Medical School

Introduction: Congenital disorder of glycosylation Ilb (CDG-Ilb) is caused by rare genetic mutations in the MOGS gene affecting mannosyl-oligosaccharide glucosidase I (glucosidase I) function. As an initial step of $\mathrm{N}$-glycan maturation, glucosidase I specifically removes the distal a-1,2-linked glucose from the protein bound $\mathrm{N}$-glycan precursor $\mathrm{Glc}_{3} \mathrm{Man}_{9} \mathrm{GlCNAC}_{2}$. Patient(s) and Methods: Here we present the clinical phenotype and the first in-depth glycomic characterization of a new female CDGllb patient. The female index case was born to non-consanguineous Caucasian parents after an uneventful pregnancy; the family history was also unrevealing. Within the first year of life, hypotonia and macroglossia accompanied by developmental delay and hearing difficulties became apparent.

Results: Trio exome analysis revealed compound heterozygosity for 2 novel mutations in the MOGS gene causing distinct single amino acid exchanges. Reduced levels of immunoglobulins were detected while further routine clinical biochemistry was unrevealing. For in-depth glycomic characterization, $\mathrm{N}$-glycans of whole serum proteins and serum-derived immunoglobulin $\mathrm{G}(\mathrm{lgG})$, transferrin (TF), and a-1-antitrypsin (AAT) as well as glycosphingolipid glycosylation of leucocytes were analyzed by multiplexed capillary gel electrophoresis coupled to laser induced fluorescence (xCGE-LIF). The glycosphingolipidome of the CDGllb patient was comparable to the controls (heterozygous parents) implying that the MOGS mutations do not affect glycosphingolipid synthesis. However, the $\mathrm{N}$-glycomic analysis by glyXbox ${ }^{\mathrm{CE}}{ }^{\mathrm{TM}}$ (glyXera, Magdeburg, Germany) revealed CDGIIb characteristic untrimmed $\mathrm{N}$-glycans $\left(\mathrm{Glc}_{3} \mathrm{Man}_{7-9} \mathrm{GlcNAc}_{2}\right)$ as well as the free tetrasaccharide $\mathrm{Glc}_{3} \mathrm{Man}$ in the CDGllb patient but not in her relatives (heterozygous parents and healthy sibling) prompting their use as diagnostic biomarkers. Furthermore, whole serum $\mathrm{N}$-glycan analysis showed reduced relative intensities of IgG-specific complex type bi-antennary $\mathrm{N}$ glycans with core-fucosylation in the CDGllb patient corroborating the diagnosed hypogammaglobulinemia. Correspondingly, increased relative intensities of sialylated bi- and tri-antennary $\mathrm{N}$-glycans, typically found on other serum proteins such as TF and AAT, were detected in whole serum of the CDGllb patient. However, specific $\mathrm{N}$-glycan profiles of serum-derived TF and AAT remained unaltered matching the unrevealing hepatic phenotype of the CDGllb patient.

Conclusion/Discussion: Taken together, the xCGE-LIF technique holds great potential as a novel diagnostic tool for the identification not only of CDG-Ilb but for other CDGs as well.

\section{P08 \\ Metachromatic leukodystrophy presenting with acute disseminated encephalomyelitis}

\author{
Meier K. ${ }^{1}$, Gärtner J. ${ }^{1}$, Huppke P. ${ }^{2}$ \\ ${ }^{1}$ Universitätsklinik Göttingen, Klinik für Kinder- und Jugendmedizin; ${ }^{2}$ Universitätsklinik \\ Jena, Klinik für Neuropädiatrie
}

Introduction: Metachromatic leukodystrophy (MLD) is an autosomal recessive neurodegenerative disorder. Defects in arylsulfatase A (ARSA) result in accumulation of sulfatides, leading to chronic progressive demyelination in the CNS and PNS. Initial symptoms of the juvenile type are ataxia, spasticity and learning or behavioral problems starting at age 4-6 years. Case report: We report on a 6-year-old girl who presented with a partial seizure. MRI showed a large right sided, contrast enhancing lesion, suggestive of acute disseminated encephalomyelitis (ADEM) or lymphoma.
Upon treatment with methylprednisolone the lesion resolved, but after 3 months a similar lesion appeared on the left side leading to right sided hemiparesis that was again responsive to steroids. However, the follow up MRI showed no new inflammatory lesions but diffuse bilateral leukodystrophy typical for MLD. As ARSA activity was only mildly reduced genetic testing was performed confirming the diagnosis. At that time the girl began to show progressive ataxia and behavioral problems.

Conclusion/Discussion: The patient described here presented with the coincides of ADEM and MLD. Interestingly, two similar cases have been reported by Anlar et al. in 2006 (Dev Med Child Neurol. 2006 May;48(5):383-7). One could speculate that ADEM might trigger the onset of MLD or that MLD predisposed to ADEM. These cases indicated that patients who present with typical ADEM but show bilateral periventricular hyperintensities on $\mathrm{T} 2$ weighted images should be investigated further for inborn leukodystrophies including MLD.

\section{P09}

Reducing the false positive rate of neonatal screening for isovaleric aciduria by a simple second-tier method

Murko S. ', Dadkhah Aseman A. ', Langrock T. ${ }^{2}$, Santer R. ${ }^{1}$

'Neonat Screen Lab, Dept Pediatr, Univ Med Cent Eppendorf, Hamburg, Germany; ${ }^{2}$ Waters GmbH, Eschborn, Germany

Introduction: Newborn screening (NBS) for isovaleric acidemia (IVA) is performed by flow injection tandem mass spectrometry (TMS) quantifying C5-carnitines (C5). Isovalerylcarnitine, however, is isobaric with pivaloylcarnitine which can be present in blood due to maternal use of pivalic acid ester-containing drugs. Antibiotics based on pivalic acid derivatives have been licensed in Germany in late 2016.

Patient(s) and Methods: During a 30-month-period (1/19-6/21), all newborns with a $\mathrm{C} 5$ concentration above cut-off $(0.5 \mu \mathrm{M})$ were included in the study. As a second-tier test, a chromatographic (UPLC C18 column) MS/MS method was applied to differentiate $C 5$ isobars. The same samples already prepared and analyzed by the NBS method were used for further differentiation of isobars. Samples of patients with genetically confirmed IVA and 2-methylbutyrylglycinuria were used for comparison.

Results: Valeryl-, Isovaleryl-, 2-methylbutyryl- and pivaloylcarnitine were well separated by our second tier test. During the study period 65 newborns out of 125000 tested had a C5 above cut-off and in all of them we detected pivaloylcarnitine as the explanation for the $\mathrm{C} 5$ elevation $(0.7$ to $8.2 \mu \mathrm{M})$. These cases showed local clusters and contact with mothers, whenever possible, confirmed intake of antibiotics at the end of pregnancy. Ten percent of these cases showed a free carnitine concentration (C0) below the reference range. No new IVA case, neither mild nor clinically relevant, was diagnosed during the study period in our lab.

Conclusion/Discussion: Discussion. Since isobaric C5-carnitines cannot be distinguished by NBSTMS, a second-tier test is important to distinguish real IVA cases from false-positive results. Inclusion of this method in NBS significantly reduces the false positive rate of IVA screening, consequently avoids further diagnostic steps and, most importantly, prevents unnecessary stress and anxiety of parents in a remarkably high number of cases. If our findings are extrapolated for all of Germany, one can assume 373 false positive cases in comparison to an average of 2 classic IVA cases per year. Conclusion: Unless licensing of pivalic acid ester-containing drugs for use during pregnancy is not reconsidered, a second-tier method for C5-carnitine determination is indispensable. 


\section{P10}

Differences of phenylalanine levels in dried blood spots and in plasma: Erythrocytes as a neglected component for this observation

Haas D. ${ }^{1}$, Hauke J. ${ }^{1}$, Schwarz K. ${ }^{1}$, Consalvi L. ${ }^{1}$, Trefz F. ${ }^{1}$, Blau N. ${ }^{2}$, Hoffmann G. ${ }^{1}$, Burgard P. ${ }^{1}$, Garbade S. ${ }^{1}$, Okun J. ${ }^{1}$

'Division of Child Neurology and Metabolic Medicine, Center for Child and Adolescent Medicine, University Hospital Heidelberg, Heidelberg, Germany; ${ }^{2}$ University Children's Hospital Zürich, Switzerland

Introduction: Monitoring phenylalanine (Phe) concentrations is critical for the management of phenylketonuria (PKU). This can be done in dried blood spots (DBS) or EDTA plasma derived from capillary or venous blood. Different techniques are used to measure Phe, the most common being flow-injection analysis tandem mass spectrometry (FIA-MS-MS) and ion exchange chromatography (IEC). Significant differences have been reported between Phe concentrations in various sample types measured by different techniques, the cause of which is not yet understood.

Patient(s) and Methods: Phe concentrations in 240 venous blood samples from 199 patients with hyperphenylalaninemia were measured in parallel in dried blood spots, EDTA plasma and erythrocytes by FIA-MS-MS and IEC.

Results: Phe concentrations were significantly lower in erythrocytes than in plasma leading to about $19 \%$ lower Phe DBS concentrations compared to plasma independent from the method used for quantification. Because variance of Phe concentrations in plasma and DBS was not linear but increased with higher concentrations a simple correction factor for converting DBS to plasma values is not sufficient. We propose quantiles regression as a method to reliably convert DBS into plasma concentrations.

Conclusion/Discussion: Lower Phe concentrations in DBS compared to plasma are mainly caused by lower erythrocyte Phe concentrations. As most therapy recommendations for PKU patients are based on plasma concentrations reliable conversion of DBS into plasma concentrations is necessary, which can be obtained by quantiles regression.

\section{P11}

\section{IEMbase: An online knowledge base and mini-expert tool for the diagnosis of inborn errors of metabolism}

Hewitson B. ${ }^{1}$, Ferreira C. ${ }^{2}$, Ounap K. ${ }^{3}$, van Karnebeek C. ${ }^{4}$, Wasserman W. ${ }^{1}$, Blau N. ${ }^{5}$

'BC Children's Hospital Research Institute, University of British Columbia, Vancouver, BC, Canada; ${ }^{2}$ National Human Genome Research Institute, National Institutes of Health, Bethesda, MD, USA; ${ }^{3}$ Department of Clinical Genetics, United laboratories, Tartu University Hospital \& Institute of Clinical Medicine, University of Tartu, Tartu, Estonia; ${ }^{4}$ Departments of Pediatrics and Clinical Genetics, Emma Children's Hospital, AUMC, Amsterdam, The Netherlands; ${ }^{5}$ Division of Metabolism, University Children's Hospital Zürich, Zurich, Switzerland

Introduction: Therapy is available to improve patient outcomes for a number of inborn errors of metabolism (IEMs); however, an accurate and timely diagnosis is required for effective treatment. This is often difficult to achieve due to a large knowledge gap between IEM specialists and the clinicians involved in early diagnoses, as well as non-specificity of symptoms for IEM disorders. The IEMbase (www.iembase.org) is an online tool aimed at bringing the knowledge of specialized experts to professionals involved in diagnoses. IEMbase consists of an expert-curated knowledge base and mini-expert diagnosis support system, which accepts a list of clinical and/ or biochemical phenotypes from the user and returns a list of matching IEM diagnoses. A mobile App for Android and iOS is available as well.

Patient(s) and Methods: The matched diagnoses are generated using a two-step algorithm to compare the input phenotypic profile with every IEM profile in the IEMbase database. The first step assesses biochemical phenotypes by calculating the cosine similarity of term frequency-inverse document frequency (TFIDF) vectors for the input profile and each IEM profile in the database. The resulting cosine similarity scores are used to rank IEM profiles in the database to determine the disorders that are to be used in the second step of the algorithm. The semantic similarity between the input clinical phenotype and IEM disorder profiles in the database are calculated in the second step of the algorithm, relying on the Human Phenotype Ontology (HPO) as the standard medical vocabulary.

Results: The disorders resulting from the mini-expert system can be used to generate differential diagnosis tables, biochemical test panels, and targeted gene panels for users to pursue further investigations for diagnosis. Approximately 400 monthly users access the information in IEMbase, currently consisting of 3430 clinical/biochemical profiles and 8465 disorder/ phenotype profiles created from 1596 IEM disorders.

Conclusion/Discussion: The IEMbase provides two key functions: first, it is a free and openly available repository of the features characterizing the different IEMs; second, it has an artificial intelligence (Al) search tool to aid the diagnosis of IEMs.

\section{P12}

Role of reactive oxygen species and protein stability of GTP cyclohydrolase in pathophysiology of Doparesponsive Dystonia (DRD)

Neumann L.', Andresen C. ${ }^{,}$Pabst A. ${ }^{1}$, Huebschmann D. ${ }^{3}$, Kuseyri Huebschmann 0. ${ }^{1}$, Richter P., , Hoffmann G. ${ }^{1}$, Opladen T.', Jung-Klawitter S. ${ }^{1}$

'University Children's Hospital Heidelberg; ${ }^{2} \mathrm{HI}-\mathrm{STEM}$ Heidelberg gGmbH, German Cancer Research Center (DKFZ); ${ }^{3}$ National Center for Tumor Diseases (NCT), German Cancer Research Center (DKFZ), Computational Oncology

Introduction: GTP cyclohydrolase I (GTPCH) is the first and rate-limiting enzyme in tetrahydrobiopterin $\left(\mathrm{BH}_{4}\right)$ biosynthesis. $\mathrm{BH}_{4}$ plays an important role in neurotransmitter synthesis as a cofactor of aromatic amino acid hydroxylases, such as tyrosine, phenylalanine and tryptophan hydroxylases. Mutations in the $\mathrm{GCH}$ gene lead to reduced $\mathrm{BH}_{4}$ bioavailability and subsequently to a lack of dopamine, serotonin, epinephrine and norepinephrine. The neurotransmitter deficiency results in a clinical picture mainly characterised by a dopa-responsive dystonia (DRD) with diurnal fluctuation.

Furthermore, $\mathrm{BH}_{4}$ is an essential cofactor for nitric oxide synthases (NOS). Consequently, reduced concentration of $\mathrm{BH}_{4}$ leads to NOS uncoupling and to increased production of reactive oxygen species (ROS).

Patient(s) and Methods: Neuronal progenitor cells (NPCs), generated from patient-specific GTPCH-deficient (GTPCHD) and wild type induced pluripotent stem cells (iPSCs), were subjected to RNA-sequencing and metabolite measurements. Data were explored using integrative bioinformatic analysis. Following the results NPCs were analysed for the expression of GTPCH, NOS and ROS levels. Moreover, overexpression of GTPCH harbouring patient-specific mutations followed by measurements of enzyme activity and stability was performed.

Results: RNA-Sequencing data and metabolite measurements revealed significantly upregulated genes involved in the generation of reactive oxygen species in GTPCHD NPCs compared to wildtype control cells. Moreover, GTPCHD NPCs were more susceptible to oxidative stress compared to healthy wild-type NPCs when challenged with the redox-cycling compound menadione or the peroxynitrite generator SIN-1. ROS and peroxynitrite scavengers, including a-tocopherol and ebselen, could only partially rescue GTPCHD NPCs. Furthermore, overexpression of mutationcarrying GTPCH proteins provided evidence for differences in enzyme activity and stability depending on the underlying mutations.

Conclusion/Discussion: The data suggest that GTPCH-deficient cells are more prone to oxidative stress. This finding can contribute to the understanding of the pathophysiology of DRD and might have implications for the therapeutic regimen. Additionally, the differences in enzyme activity and stability depending on the underlying mutations might purvey an explanation for the phenotypic differences observed in patients. 
P13

\section{Treating neutropenia and neutrophil dysfunction in glycogen storage disease type lb with an SGLT2 inhibitor}

Wortmann S. ${ }^{1}$, Lagler F. ${ }^{1}$, Derks T. ${ }^{2}$, van Hove J. ${ }^{3}$, Mayr J. ${ }^{1}$

'University Children's Hospital, Paracelsus Medical University (PMU), Salzburg, Austria ${ }^{3}$ Section of Metabolic Diseases, Beatrix Children's Hospital, University Medical Center Groningen, University of Groningen, Groningen, The Netherlands; ${ }^{4}$ University Children's Hospital, Colorado, USA

Introduction: Neutropenia and neutrophil dysfunction cause serious infections and inflammatory bowel disease in glycogen storage disease type Ib (GSD-lb). The discovery that accumulating 1,5-anhydroglucitol6-phosphate (1,5AG6P) caused neutropenia in a glucose-6-phosphatase 3 (G6PC3)-deficient mouse model and in 2 rare diseases (GSD-lb and G6PC3 deficiency) led us to repurpose the widely used antidiabetic drug empagliflozin, an inhibitor of the renal glucose cotransporter sodium glucose cotransporter 2 (SGLT2).

Patient(s) and Methods: Off-label use of empagliflozin in 4 GSD-lb patients with incomplete response to granulocyte colony-stimulating factor (GCSF) treatment

Results: Empagliflozine treatment decreased serum 1,5AG and neutrophil 1,5AG6P levels within 1 month. Clinically, symptoms of frequent infections, mucosal lesions, and inflammatory bowel disease resolved, and no symptomatic hypoglycemia was observed. GCSF could be discontinued in 2 patients and tapered by $57 \%$ and $81 \%$, respectively, in the other 2 . The fluctuating neutrophil numbers in all patients were increased and stabilized. We further demonstrated improved neutrophil function: normal oxidative burst (in 3 of 3 patients tested), corrected protein glycosylation ( 2 of 2), and normal neutrophil chemotaxis (1 of 1), and bactericidal activity (1 of 1) under treatment.

Conclusion/Discussion: In conclusion, the glucose-lowering SGLT2 inhibitor empagliflozin, used for type 2 diabetes, was successfully repurposed for treating neutropenia and neutrophil dysfunction in the rare inherited metabolic disorder GSD-Ib without causing symptomatic hypoglycemia. We ascribe this to an improvement in neutrophil function resulting from the reduction of the intracellular concentration of 1,5AG6P.

\section{P14}

\section{Health outcomes of infants with vitamin B12 deficiency identified by newborn screening and early treated}

Mütze U. ', Walter M. ', Keller M. ', Gramer G. ', Garbade S. ', Florian G. ${ }^{1}$, Haas D. ${ }^{1}$ Posset R. ', Grünert S. ${ }^{2}$, Hennermann J. ${ }^{3}$, Thimm E. ${ }^{4}$, Fang-Hoffmann J.', Syrbe S. ${ }^{5}$, Okun J. ${ }^{1}$, Hoffmann G. ${ }^{1}$, Kölker S. ${ }^{1}$

'Division of Child Neurology and Metabolic Medicine, Center for Child and Adolescent Medicine, University Hospital Heidelberg, Heidelberg, Germany; ${ }^{2}$ Department of General Pediatrics, Adolescent Medicine and Neonatology, Medical Center - University of Freiburg, Faculty of Medicine, Freiburg, Germany; ${ }^{3}$ Villa Metabolica, Department of Pediatric and Adolescent Medicine, Mainz University Medical Center, Mainz, Germany; ${ }^{4}$ Department of General Pediatrics, Neonatology, and Pediatric Cardiology, University Children's Hospital, Heinrich Heine University Düsseldorf, Düsseldorf, Germany; ${ }^{5}$ Division of Pediatric Epileptology, Center for Child and Adolescent Medicine, University Hospital Heidelberg, Heidelberg, Germany

Introduction: Newborn screening (NBS) for vitamin $\mathrm{B}_{12}$ deficiency was shown to be feasible, identifying an unexpectedly high birth prevalence between 3-19 in 100,000 (i.e. 1 in 30,000-5,355) newborns. The aim of this study was to evaluate the clinical and cognitive outcomes at age $1.5 \pm 0.5$ years of infants with vitamin $B_{12}$ deficiency identified by NBS and early treated.

Patient(s) and Methods: Prospective multi-center observational study on health outcomes of 31 infants with vitamin $B_{12}$ deficiency identified by NBS. Neurodevelopment was assessed by Denver Developmental Screening Test (DDST).
Results: In 285,862 newborns screened between 2016 to 2019, the estimated birth prevalence of vitamin $B_{12}$ deficiency was 26 in 100,000 newborns, with high seasonal variations (lowest in summer: 8 in 100,000). Infants participating in the outcome study $(N=31)$ were supplemented with vitamin $B_{12}$ for a median (range) of 5.9 (1.1-16.2) months. All achieved age-appropriate test results in DDST at age $15(11-23)$ months and did not present with symptoms characteristic for vitamin $B_{12}$ deficiency. Most $(81 \%, N=25)$ mothers of affected newborns had a hitherto undiagnosed (functional) vitamin $B_{12}$ deficiency, and, subsequently, received specific therapy.

Conclusion/Discussion: Neonatal vitamin $\mathrm{B}_{12}$ deficiency can be screened by NBS, preventing the manifestation of irreversible neurological symptoms and the recurrence of vitamin $B_{12}$ deficiency in future pregnancies through adequate treatment of affected newborns and their mothers. The high frequency of mothers with migrant background having a newborn with vitamin $B_{12}$ deficiency highlights the need for improved prenatal care.

\section{P15}

\section{Early prediction of disease severity in cytosolic Urea Cycle Disorders}

Zielonka M. ', Scharre S. ', Seidl M. ', Kölker S. ', Gleich F. ', Stützenberger N. ${ }^{1}$ Probst J.', Druck A. ', Nagamani S. ', Gropman A. ${ }^{3}$, Hoffmann G. ', Garbade S. ', Posset R.

${ }^{1}$ Center for Pediatric and Adolescent Medicine, Division of Pediatric Neurology and Metabolic Medicine, University Hospital Heidelberg, Heidelberg, Germany; ${ }^{2}$ Department of Molecular and Human Genetics, Baylor College of Medicine and Texas Children's Hospital, Houston, Texas, USA; ${ }^{3}$ Children's National Health System and The George Washington School of Medicine, Washington, District of Columbia, USA

Introduction: Citrullinemia type 1 (CTLN1) and argininosuccinic aciduria (ASA) are inherited urea cycle disorders (UCDs) with highly variable clinical phenotypes. Since the phenotypic severity has not been reliably predictable early during the disease course so far, we aimed to design a reliable disease prediction model.

Patient(s) and Methods: We used a newly established mammalian biallelic expression system to determine residual enzymatic activity of argininosuccinate synthetase 1 (ASS1) and argininosuccinate lyase (ASL) in 129 individuals with CTLN1 and ASA. Residual enzymatic ASS1 and ASL activities were correlated to standardized biochemical and clinical outcome parameters available from the UCDC and E-IMD databases.

Results: Residual enzymatic ASS1 and ASL activity correlated with peak plasma ammonium concentration at the initial hyperammonemic episode (HAE). Individuals with $\leq 8 \%$ or $9 \%$ of residual enzymatic ASS1 or ASL activity, respectively, developed more frequent and more severe HAEs during their disease course than individuals with values above these thresholds. Long-term cognitive outcome was associated with the degree of enzymatic dysfunction, highlighting that residual enzymatic activities of UCD enzymes allow a reliable disease severity prediction.

Conclusion/Discussion: Residual enzymatic ASS1 and ASL activity reliably predict the phenotypic severity in CTLN1 and ASA, thereby qualifying as predictive biomarkers for the disease course and neurocognitive outcome. The established disease prediction models will serve as important tools for the counseling of afflicted individuals and their families as well as for the evaluation of existing (e.g. newborn screening) or potential novel diagnostic and therapeutic approaches in the future. 


\section{P16}

Long-term impact of medical management on growth and weight in individuals with urea cycle disorders

Posset R. ${ }^{1}$, Garbade S. ${ }^{1}$, Gleich F. ${ }^{1}$, Gropman A. ${ }^{2}$, de Lonlay P. ${ }^{3}$, Hoffmann G. ${ }^{\prime}$, García-Cazorla Á. ${ }^{4}$, Nagamani S. ${ }^{5}$, Baumgartner M. ${ }^{6}$, Schulze A. ${ }^{7}$, Dobbelaere D. , Yudkoff M. ${ }^{9}$, Scharre S. ', Probst J.', Seidl M. ', Druck A.', Kölker S. ' , Zielonka M. ${ }^{1}$

${ }^{1}$ Center for Pediatric and Adolescent Medicine, Division of Pediatric Neurology and Metabolic Medicine, University Hospital Heidelberg, Germany; ${ }^{2}$ Children's National Health System and The George Washington School of Medicine, Washington, District of Columbia, USA; ${ }^{3}$ Hôpital Necker-Enfants Malades, Assistance Publique-Hôpitaux de Paris, Service de Maladies Metaboliques (MaMEA), Université Paris-Descartes, Paris, France; ${ }^{4}$ Hospital San Joan de Deu, Institut Pediàtric de Recerca. Servicio de Neurologia and CIBERER, ISCIII, Barcelona, Spain; ${ }^{5}$ Department of Molecular and Human Genetics, Baylor College of Medicine and Texas Children's Hospital, Houston, TX, USA; ${ }^{\circ}$ University Children's Hospital Zurich and Children's Research Center, Zurich, Switzerland; ${ }^{7}$ University of Toronto and the Hospital for Sick Children, Toronto, ON, Canada; ${ }^{8}$ Centre de Référence Maladies Héréditaires du Métabolisme de L'Enfant Et de L'Adulte, Jeanne de Flandre Hospital, CHRU Lille, and Faculty of Medicine, University Lille 2, Lille, France; ${ }^{9}$ School of Medicine and Children's Hospital of Philadelphia, University of Pennsylvania, Philadelphia, PA, USA

Introduction: Low protein diet and sodium or glycerol phenylbutyrate, two pillars of recommended long-term therapy for individuals with urea cycle disorders (UCDs), involve the risk of iatrogenic growth failure. Limited evidence-based studies hamper our knowledge on the long-term effects of the proposed medical management in individuals with UCDs.

Patient(s) and Methods: We studied the impact of medical management on growth and weight development in 307 individuals longitudinally followed by UCDC and the E-IMD Consortia Study Group.

Results: Symptomatic individuals were at risk of progressive growth retardation independent from the underlying disease and the degree of natural protein restriction. Growth impairment was determined by disease severity and associated with reduced or borderline plasma branched-chain amino acid (BCAA) concentrations. In contrast however, weight development remained unaffected. Interestingly, liver transplantation restored BCAA levels and appeared to have a beneficial effect on growth.

Conclusion/Discussion: Progressive growth impairment depends on disease severity and plasma BCAA concentrations, and appears not to be an effect of iatrogenic protein restriction. Future clinical trials are necessary to evaluate whether supplementation with BCAAs might improve growth in UCDs.

\section{P17}

\section{The clinical spectrum and molecular heterogeneity of Succinyl-CoA:3-oxoacid coenzyme A transferase (SCOT) deficiency}

Grünert S. ${ }^{1}$, Foster W. ${ }^{2}$, Schumann A. ${ }^{1}$, Allan L. ${ }^{3}$, Pontes C. ${ }^{4}$, Roloff S. ${ }^{5}$, Weinhold N. ${ }^{5}$, Yue W. ${ }^{2}$, AlAsmari A. ${ }^{6}$, Obaid O. ${ }^{7}$, Faqeih E. ${ }^{7}$, Stübbe L. ${ }^{8}$, Yamamoto R. ${ }^{8}$, Gemperle-Britschgi C. ${ }^{9}$, Walter M. ', Spiekerkoetter U. ', Mackinnon S. ${ }^{2}$, Sass J. ${ }^{10}$

'Department of General Paediatrics, Adolescent Medicine and Neonatology, Medical Center - University of Freiburg, Faculty of Medicine, Freiburg, Germany; ${ }^{2}$ Nuffield Department of Medicine, University of Oxford, Oxford United Kingdom; ${ }^{3}$ Departments of Paediatrics and Clinical Genetics, Copenhagen University Hospital, Rigshospitalet, Copenhagen, Denmark; ${ }^{4}$ Centre for Paediatrics and Adolescent Medicine, Heidelberg University Hospital, Heidelberg, Germany; ${ }^{5}$ Charité - Universitätsmedizin Berlin, Corporate Member of Freie Universität Berlin, Humboldt-Universität zu Berlin, and Berlin Institute of Health, Center for Chronically Sick Children, Berlin, Germany; ${ }^{6}$ Children's Specialist Hospital, King Fahad Medical City, Riyadh, Kingdom of Saudi Arabia; ${ }^{7}$ King Fahad Medical City, Riyadh, Kingdom of Saudi Arabia; ${ }^{8}$ MVZ Dr. Eberhard \& Partner Dortmund GbR (ÜBAG), Dortmund, Germany; ${ }^{\circ}$ University Children's Hospital, Clinical Chemistry \& Biochemistry and Children's Research Center, Zürich, Switzerland; ${ }^{10}$ Research Group Inborn Errors of Metabolism, Department of Natural Sciences \& Institute for Functional Gene Analytics (IFGA), Bonn-Rhein-Sieg University of Applied Sciences, Rheinbach
Introduction: Succinyl-CoA:3-oxoacid coenzyme A transferase deficiency (SCOTD) is a rare autosomal recessive disorder of ketone body utilization caused by biallelic OXCT1 variants. Less than 35 patients with this metabolic disease have been reported so far.

Patient(s) and Methods: In order to characterize the clinical and genetic spectrum of SCOTD, we performed a systematic literature search. 34 patients with genetically confirmed SCOTD and available clinical description were identified by this approach. Another 10 unpublished cases were added to this analysis. Data were analyzed with the focus on clinical course, neurologic outcome, biochemical and genetic findings. Structural mapping and in silico analysis of protein variants were performed.

Results: All patients presented with severe ketoacidotic episodes between $36 \mathrm{~h}$ and 3 years of age (median age at initial presentation 7 months). The majority of patients (70\%) manifested in the first year of life, thereof about one quarter within the neonatal period. Two cases were fatal, while all other patients $(95 \%)$ were alive at the time of the report. The neurologic outcome of surviving individuals was favourable with $92 \%$ of patients showing normal development. OXCT1 variants reported in literature comprise 29 missense variants, five nonsense variants, two insertions, and one large deletion. Using the crystal structure of the human SCOT enzyme, missense variants were analyzed by both sequence-based and structure-based methods to gain insights into their biochemical consequences. Pathogenic variants cluster in SCOT protein regions that affect certain structures of the protein. The described pathogenic variants can be viewed in an interactive map of the SCOT protein at https://michelanglo. sgc.ox.ac.uk/r/oxct.

Conclusion/Discussion: This comprehensive overview of all reported cases of SCOTD has helped to characterize the clinical spectrum of this rare ketolysis defect. Although SCOTD is a rather benign disorder and most patients show a favourable outcome, severe and fatal cases have been reported. As the diagnosis requires enzyme studies or mutation analysis, SCOTD may be underdiagnosed (Grünert et al., Biochimie 2021; 183:55$62)$.

\section{P18}

Peripheral neuropathy in LCHAD and MTP deficiency

Grünert S. ${ }^{1}$, Eckenweiler M. ${ }^{2}$, Haas D. ${ }^{3}$, Lindner M. ${ }^{4}$, Tsiakas K. ${ }^{5}$, Santer R. ${ }^{5}$, Tucci S. ${ }^{6}$, Spiekerkoetter U. ${ }^{6}$

'Department of General Paediatrics, Adolescent Medicine and Neonatology, Medical Centre - University of Freiburg, Faculty of Medicine, Freiburg, Germany; ${ }^{2}$ Department of Neuropediatrics and Muscle Disorders, University Medical Center, Faculty of Medicine, University of Freiburg, Germany; ${ }^{3}$ Department of Neuropaediatrics and Paediatric Metabolic Medicine, Center for Child and Adolescent Medicine, University Hospital Heidelberg, Heidelberg, Germany; ${ }^{4}$ Department of Paediatric Neurology, University Children's Hospital, Frankfurt/Main, Germany; ${ }^{5}$ University Children's Hospital, University Medical Centre Hamburg-Eppendorf, Hamburg, Germany; ${ }^{6}$ Department of General Paediatrics, Adolescent Medicine and Neonatology, Medical Centre - University of Freiburg, Faculty of Medicine, Freiburg, Germany

Introduction: Long-chain 3-hydroxyacyl-CoA dehydrogenase deficiency (LCHADD) and mitochondrial trifunctional protein deficiency (MTPD) are two inherited disorders of mitochondrial long-chain fatty acid oxidation with peripheral neuropathy as a common long-term complication. The underlying pathophysiology of neuropathy in LCHADD/MTPD is still unknown.

Patient(s) and Methods: We report neurological findings and electrophysiological studies in a series of 8 LCHAD deficient and 11 MTP deficient patients. The diagnosis was confirmed genetically in all patients. Retrospective information was collected from medical records.

Results: The age of the patients ranged between 0.5 and 25 years with a median age of 8.0 years. Neuropathy was found in $58 \%$ of patients with a slightly higher prevalence in MTPD compared to LCHADD (70\% versus $50 \%$, respectively). MTPD patients also showed a significantly earlier onset of neuropathy compared to LCHADD patients (median age at onset 4.7 versus 15.3 years, respectively, $p=0.047$ ). 
In four patients missed by newborn screening, isolated peripheral neuropathy was the first and only presenting symptom. Forty-five percent of patients showed a sensorimotor neuropathy, whereas $27 \%$ had a pure motor and $27 \%$ an isolated sensory form. In 4 patients with an acute onset or aggravation of neuropathy following specific triggers, neuropathic symptoms were at least partly reversible.

Conclusion/Discussion: Peripheral neuropathy is a long-term complication of LCHADD and MTPD that cannot be fully prevented with current treatment strategies despite early diagnosis by newborn screening and early initiation of therapy. Electrophysiological studies show that LCHADD/MTPD neuropathy is typically an axonal neuropathy with secondary demyelination. Although neuropathy is usually considered irreversible, our observations indicate that neuropathic symptoms can be partly reversible in patients with acute onset or aggravation of neuropathy. Further research is needed to elucidate the underlying factors causing axonal damage in LCHADD/MTPD in order to identify new therapeutic targets.

\section{P19}

\section{Frequent sequence variants of human glycine $\mathrm{N}$-acyltransferase (GLYAT) and inborn errors of metabolism}

\section{Schulke D. ${ }^{1}$, Sass J.1}

'Research Group Inborn Errors of Metabolism, Department of Natural Sciences, BonnRhein-Sieg University of Applied Sciences, Rheinbach, Germany

Introduction: Human GLYAT is a phase II enzyme which is involved in the elimination of various xenobiotics. Due to coenzyme A ( $\mathrm{coA}$ ) activation GLYAT also affects coA homeostasis and may contribute to the elimination of coA esters which accumulate in several inborn errors of metabolism. Patient(s) and Methods: Albeit only few sequence variants of GLYAT gene have been reported so far, two studies described overexpression of the human protein with the reference sequence ("wild-type") and sequence variants in bacterial system with partial enzyme characterization. Referring to that we reevaluated the wild-type enzyme and the two sequence variants p.(Gln61Leu) and p.(Asn156Ser)-variant p.(Gln61Leu) had not been subjected to functional characterization before, $p$.(Asn156Ser) may be the authentic wild-type of human GLYAT due to higher prevalence and enzyme activity compared to the canonical wild-type. We performed analyses in GLYAT purified from the E.coli strain Origami 2(DE3) and in homogenates of overexpressing HEK293 cells. Beyond that we reexamined intracellular localization of human GLYAT in HEK293 cells using stable expressed GLYATeGFP fusion protein and confocal microscopy.

Results: Purified wild-type GLYAT resulted in $\mathrm{v}_{\max }$ of $490 \pm 274 \mathrm{U} / \mathrm{mg}$ protein; $p$.(GIn61Leu) variant yielded merely $20 \%$ of wild-type activity while specific activity of p.(Asn156Ser) was threefold increased. This activity ratio was not observed with HEK293 cell homogenates which showed similar maximum activities of $\mathrm{v}_{\max }$ of 39 and $74 \mathrm{U} / \mathrm{mg}$ protein for wild-type GLYAT. км values of E.coli enzyme $(79 \pm 38 \mu \mathrm{mol} / \mathrm{L})$ resembled those of HEK293 cell homogenates ( 160 and $56 \mu \mathrm{mol} / \mathrm{L}$ ). Confocal microscopy confirmed intramitochondrial localization of human wild-type GLYAT in the HEK293 cells.

Conclusion/Discussion: Variant p.(Gln61Leu) does not only impair GLYAT activity in vitro, it is also prevalent in Caucasian Afrikaner cohort in South Africa. Due to prevalence of isovaleric acidemia disease potential pharmacogenetic implications may arise. Thus, studies going beyond the report by Schulke \& Sass (Biochimie 2021;183:30-34) are indicated.
P20

Persistent effect of arimoclomol in patients with Niemann-Pick disease type C: 24-month results from an open-label extension of a pivotal Phase 2/3 study

Patterson M. ${ }^{1}$, Mengel E. ${ }^{2}$, Da Riol R. ${ }^{3}$, Del Toro M. ${ }^{4}$, Deodato F. ${ }^{5}$, Gautschi M. ${ }^{6}$, Grunewald S. ${ }^{7}$, Grønborg S. ${ }^{8}$, Harmatz P. ${ }^{9}$, Héron B. ${ }^{10}$, Maier E. ${ }^{11}$, Roubertie A. ${ }^{12}$, Santra S. ${ }^{13}$, Tylki-Szymańska A. ${ }^{14}$, Day S. ${ }^{15}$, Andreasen A. ${ }^{16}$, Aavang Geist M. ${ }^{16}$, Havnsøe Torp Petersen N. ${ }^{16}$, Ingemann L. ${ }^{16}$, Hansen T. ${ }^{16}$

'Mayo Clinic, Rochester, MN, United States; ${ }^{2}$ SphinCS GmbH, Hochheim am Main, Germany; ${ }^{3}$ Academic Hospital, Santa Maria della Misericordia', Udine, Italy; ${ }^{4}$ Vall d'Hebron University Hospital, Barcelona, Spain; ${ }^{5}$ Ospedale Pediatrico Bambino Gesù, IRCCS, Rome, Italy; ${ }^{6}$ Department of Pediatrics and Institute of Clinical Chemistry, Inselspital, University Hospital Bern, Bern, Switzerland; ' $U C L$ Great Ormond Street Institute of Child Health, National Institute for Health Research Biomedical Research Centre, London, United Kingdom; ${ }^{8}$ Copenhagen University Hospital, Copenhagen, Denmark; ${ }^{\circ}$ UCSF Benioff Children's Hospital Oakland, Oakland, CA, United States; ${ }^{10}$ Reference Centre for Lysosomal Diseases, University Hospital Armand Trousseau, Paris, France; ${ }^{1}$ University of Munich Children's Hospital, Munich, Germany; ${ }^{12}$ Centre Hospitalier Universitaire de Montpellier, Montpellier, France; ${ }^{13}$ Birmingham Children's Hospital, Birmingham, United Kingdom; ${ }^{14} \mathrm{Children's}$ Memorial Health Institute, Warsaw, Poland; ${ }^{15}$ Clinical Trials Consulting \& Training Limited, Buckingham, United Kingdom; ${ }^{16}$ Orphazyme A/S, Copenhagen, Denmark

Introduction: Niemann-Pick disease type C (NPC), a rare, progressive neurodegenerative disease, has considerable unmet need. The 12-month, double-blind (DB), placebo-controlled, Phase 2/3 NPC-002 study suggested a clinically meaningful effect of arimoclomol on NPC progression. We present interim results of a 24-month open-label extension (OLE), providing efficacy and safety data for arimoclomol up to Month 36 (M36).

Patient(s) and Methods: All OLE patients received arimoclomol; routine clinical care, including miglustat, was maintained. The primary endpoint was change in disease severity based on 5-domain NPC Clinical Severity Scale (5D-NPCCSS) scores from baseline to Months 18, 24, 30 and 36. Subgroups comprised participants aged $\geq 4$ years, treated with miglustat and without double functional null mutations.

Results: Forty-one participants entered the OLE (arimoclomol, $n=26$; placebo, $n=15)$; 33 completed M36. At M36, sustained benefit of arimoclomol was observed on the 5D-NPCCSS, with a mean (SD) change from baseline of 3.5 (5.6) for the arimoclomol-arimoclomol group (3 years' arimoclomol treatment; DB and OLE) and 0.9 (2.1) for the placebo-arimoclomol group (2 years' arimoclomol treatment; OLE only). In comparison, NPC progression (SE) with only standard of care was 5.2 (1.41) after 3 years and 3.5 (0.94) after 2 years, as estimated by extrapolation from NPC-001 observational and NPC-002 placebo data. A consistent benefit of sustained arimoclomol treatment was observed across subgroups at M36. The proportion of adverse events at M12-24 (87.8\%) and M24-36 (82.9\%) were similar to the DB phase ( $88.2 \%)$.

Conclusion/Discussion: Arimoclomol provided sustained treatment effect over 36 months; it was well tolerated, with a consistent safety profile.

\section{P21}

Biallelic variants in GZF1 and VPS45 lead to Larsen syndrome and Congenital Disorders of Glycosylation (CDG)

Falkenstein K. ${ }^{1}$, Hutter M. ${ }^{1}$, Dimitrov B. ${ }^{1}$, Himmelreich N. ${ }^{1}$, Hecker A. ${ }^{1}$, Stahl C. ${ }^{1}$, Schuler E. ${ }^{1}$, Bürger F. ${ }^{1}$, Kölker S. ${ }^{~}$, Kornak U. ${ }^{2}$, Thiel C. ${ }^{1}$

'Zentrum für Kinder- und Jugendmedizin, Kinderheilkunde I, Heidelberg; ${ }^{2}$ Charité, Institut für Medizinische Genetik und Humangenetik, Berlin

Introduction: We here present data of an eight-year-old female patient who suffers from small stature, hyperlordosis, joint abnormalities, pes planovalgus as well as skewed, sunken and splayed feet. Further symptoms include craniosynostosis with oxycephalus, midface hypoplasia, exophthalmos and dental crowding. She also presents with global develop- 
mental delay, conductive hearing loss and vision abnormalities. Notably, the clinical symptoms did not appear until her first year of life.

Patient(s) and Methods: Diagnostics for Congenital Disorders of Glycosylation (CDG) by isoelectric focusing of transferrin and apolipoprotein CIII showed a combined N- (CDG-II) and O-glycosylation deficiency. Further, total serum glycome analysis by mass spectrometry mainly revealed a loss of galactose and sialic acid residues on N-glycans.

Results: Whole Exome Sequencing identified a homozygous variant (p.Glu289*) in the GDNF-inducible zinc finger protein 1 (GZF1), which is known to lead to 'Larsen syndrome', a disease that is characterized by e. $\mathrm{g}$. bone, joint and facial abnormalities, but is not linked to CDG. Interestingly, also a homozygous variant (p.Ala392Thr) in the vacuolar sorting protein 1 homolog (VPS45) was detected. VPS45 is associated with subunits of the Conserved Oligomeric Golgi (COG) complex, whose malfunction leads to abnormal intra Golgi protein sorting and subsequently to severe $\mathrm{N}$ - and $\mathrm{O}$ hypoglycosylation. Further analysis by qRT-PCR, Western blot and immunofluorescence studies showed deregulations of the transcript and protein levels, as well as delocalization of GZF1 and VPS45 within the patient fibroblasts.

Conclusion/Discussion: Since also the assembly of the COG complex was affected, we assume that the severe multisystem phenotype of our patient is caused due to biallelic variants in GZF1 and VPS45, whereby the Larsen Syndrome and CDG are linked for the very first time.

\section{P22}

\section{AMPA receptor dysregulation as a possible cause of stiff- baby-syndrome}

\section{Kaiser N. ${ }^{1}$, Weichselbaum A. ${ }^{2}$, Magg J. ${ }^{1}$, Haack T. ${ }^{3}$, Bevot A. ${ }^{1}$}

'Department of Pediatric Neurology, University Children's Hospital, Tuebingen, Germany; 2Department of Pediatric Neurology, Kantonsspital Graubuenden, Chur, Switzerland; ${ }^{3}$ Institute of Medical Genetics and Applied Genomics, University of Tuebingen, Germany

Introduction: We report on two affected boys with clinical features of extreme stiff-baby-syndrome born to healthy consanguineous parents.

Patient(s) and Methods: Patient $1\left({ }^{*} 11.2015\right)$ presented immediately postnatally with overexcitability and extreme rigor, leading to feeding and breathing difficulties. Extensive diagnostic workup including biochemical studies, metabolic screening, electrophysiology and MRI did not show pathological results. An initial exome analysis within a scientific research program remained inconclusive. Sedative and antispasmodic agents were given as symptomatic and probatory medication, followed by diverse other medication, as a neurotransmitter disorder had been suspected. The medical treatment did not lead to a relevant improvement, the severe muscular hypertonia was progressive, the stiffness even led to fractures. The boy died due to breathing problems within 8 months.

Patient $2\left({ }^{*} 01.2020\right)$ became symptomatic a few days after birth, showing an equal clinical picture. As it was considered to be the same disease, the examinations done in his brother with negative results were not repeated, but further complementary neurometabolic and genetic testing was conducted.

Results: Trio exome analysis (patient 2 and parents) revealed a homozygous C.162G $>C$ variant in ATAD1 predicted to interfere with correct splicing. The same homozygous change was consecutively found in preserved fibroblasts of patient 1 .

In 2018 three cases of stiff-baby-syndrome with homozygous mutations in ATAD1 were published. It was discussed that an ATAD1 mutation with loss of function would result in elevated postsynaptic AMPA receptor expression and amplification of the excitatory glutamate system. Therefore, the AMPA receptor antagonist Perampanel was considered to be possibly effective and was given to patient 1 . The boy benefited concerning the muscular hypertonia and was more relaxed, so that the sedative and analgetic medication could be reduced. Nevertheless, he died at the age of 7 months due to pneumonia in palliative care.

Conclusion/Discussion: The meticulous description of cases and family histories, combined with reevaluation of genetic data and the detailed re- view of literature, as well as preservation of material like blood or fibroblasts for later testing is needed to solve at least some rare neurological diseases. Especially as in disorders of neurotransmission there might be individual drug options.

\section{P23}

\section{Analysing immune response to enzyme replacement therapy in patients with CLN2 disease}

Westermann L.', Schmidtke C. ', Schneider E.', Schwering C.' , Wibbeler E.', Nickel M. ${ }^{1}$, Tolosa E. ${ }^{1}$, Schulz A. ${ }^{1}$

'Department of Pediatrics, University Medical Center Hamburg-Eppendorf, Hamburg, Germany,

Introduction: Neuronal ceroid-lipofuscinosis type 2 (CLN2) disease is a neurodegenerative lysosomal storage disorder caused by mutations in the TPP1 gene. Affected children show first clinical symptoms between 1.5 and 4 years of age, including psychomotor decline, seizures and loss of vision.

Currently, intraventricular enzyme replacement therapy (ICV-ERT) with recombinant human TPP1 (cerliponase alfa) is the only approved treatment. It was shown to slow down the loss of motor and language function. Like in other ERT, immune reaction to the recombinant enzyme is a common adverse event and increases the risk of production of neutralising antibodies and subsequently loss of treatment efficacy. Therefore, it is important to better predict and prevent adverse immunological reactions to the enzyme.

Patient(s) and Methods: We followed 33 CLN2 patients undergoing ICV-ERT at our centre. The cohort consists of 13 male and 20 female patients with mean age at therapy start of $68.4 \pm 29.3$ months. In these patients, we collected clinical data on immune reaction and complemented these data with analysis of serum and cerebrospinal fluid (CSF). In a subset of twelve patients, we determined the CRIM-status (cross-reactive immunological material) and correlated it with the risk of an immunological reaction.

Results: Twenty-five out of 33 patients showed an immunological reaction to ICV-ERT. Common symptoms were fever, tachycardia, nausea and vomiting, seizures and somnolence. CSF samples obtained routinely prior to start of enzyme infusion did not reveal any changes to predict an immune reaction.

Analysis of the CRIM-status showed that nine out of twelve patients were CRIM negative and of these, seven presented with an immune response to the enzyme. However, all three patients who were CRIM positive had an immune reaction as well.

Conclusion/Discussion: Immunological reactions to the ICV-ERT are common side effects in patients with CLN2 disease independent of CRIMstatus. Results from this study will improve understanding of immune reactions to the recombinant enzyme. This is crucial because enzyme replacement therapy, and also future gene therapy approaches, can be complicated by hypersensitivity responses toward the therapeutic enzyme that may render the treatment ineffective. 


\section{P24}

\section{SLC35A2-CDG can also be associated with a mild clinical} phenotype in combination with autism spectrum disorders

Hecker A. ${ }^{1}$, Stahl C. ${ }^{1}$, Falkenstein K. ${ }^{1}$, Himmelreich N. ', Dimitrov B. ', Geiger V. ${ }^{1}$, Thiel G. ${ }^{2}$ Thiel $C .{ }^{1}$

${ }^{1}$ Center for Child and Adolescent Medicine, University Hospital Heidelberg, Heidelberg, Germany; ${ }^{7}$ Praxis für Humangenetik, Berlin, Germany

Introduction: Congenital disorders of glycosylation (CDG) comprise a continuously growing group of monogenetic inherited diseases in glycoconjugate biosynthesis. By this, CDG leads to failure of physiological glycosylation of proteins and lipids by covalently attached oligosaccharides. The molecular defect of SLC35A2-CDG is caused by variants in the X-chromosomal gene for the Golgi UDP-galactose transporter (SLC35A2). SLC35A2 transports UDP-galactose from the cytosol into the Golgi apparatus, where the nucleotide-activated sugar is used as donor sugar substrate for the synthesis of complex and hybrid-type $\mathrm{N}$-glycans. Patients commonly show a prominent CDG-II profile by isoelectric focusing (IEF) of transferrin and a severe clinical phenotype which is characterized by e. g. macrocephaly, mental retardation, severe myelination delay, epileptic encephalopathy, global developmental delay, muscular hypotonia, shortened extremities and dysmorphic facial features.

Patient(s) and Methods: N-glycans of transferrin and of whole serum glycoproteins were analysed by isoelectric focusing and mass spectrometry, respectively. Variant analysis was conducted by WES and confirmed by Sanger sequencing. Oral galactose treatment was conducted with $1 \mathrm{~g}$ galactose/kg bodyweight/day for 15 months.

Results: Here we present new data of a female SLC35A2-CDG-patient carrying a de novo heterozygous variant (c.1A>G; p.M1V) and presenting with an autism spectrum disorder but a significantly milder clinical phenotype of biochemical and clinical parameters than other SLC35A2-CDG-patients reported before. Treatment with oral galactose supplementation improved cognitive and motoric skills, although the glycosylation pattern of transferrin and of whole serum glycoproteins did not reach normal values. Conclusion/Discussion: In conclusion, we expand the knowledge on SLC35A2-CDG and the therapeutic approach by oral galactose supplementation Furthermore, we suggest adding SLC35A2 to gene panels for autism spectrum disorders.

\section{P25}

Missense variant c.1460T>C (p.L487P) enhances protein degradation of ER mannosyltransferase ALG9 in two new ALG9-CDG patients presenting with West-Syndrome and hypsarrhythmia

Himmelreich N. ${ }^{1}$, Dimitrov B. ${ }^{1}$, Geiger V. ${ }^{1}$, Zielonka M. ${ }^{1}$, Hutter M. ${ }^{1}$, Hüllen A. ${ }^{1}$, Beedgen L. ${ }^{1}$, Hoffmann G. ${ }^{1}$, Juenger H. ${ }^{2}$, Müller H. ${ }^{2}$, Lorenz I. ${ }^{3}$, Busse B. ${ }^{4}$ Marschall C. ${ }^{4}$, Schlüter G. ${ }^{5}$, Thiel C. ${ }^{1}$

${ }^{1}$ Centre for Child and Adolescent Medicine, Pediatrics I, University of Heidelberg; ${ }^{2}$ Kinderabteilung Klinikum Kempten; ${ }^{3}$ Klinik für Kinder und Jugendliche, Abteilung fuer Neuropaediatrie und Sozialpaediatrie, Universitaet Erlangen; ${ }^{4} \mathrm{MVZ}$ Martinsried, Martinsried; ${ }^{5}$ Praenatalmedizin, Gynaekologie und Genetik, Nuernberg

Introduction: Mannosyltransferase ALG9 is a twostep catalysing glycosyltransferase working on the luminal side of the ER membrane. Deficiency of this enzyme leads to ALG9-CDG, a CDG-I disorder within the group of Congenital Disorders of Glycosylation (CDG). We here present the clinical symptoms of two new and unrelated ALG9-CDG patients and describe the biochemical effects of the genetic variant on protein level.

Patient(s) and Methods: N-glycosylation was addressed by isoelectric focusing (IEF) of serum transferrin and by mass spectrometry (MS) of N-glycans released from whole serum glycoproteins. Lipid-linked oligosaccharide (LLO) analysis, ALG9 expression studies by qRT-PCR and Western blot as well as protein stability assays were conducted in patient-derived skin fibroblasts. Variant analysis was carried out by Sanger sequencing in case of patient 1 and by WES in case of patient 2 .

Results: Both patients presented with global developmental delay, psychomotor retardation, facial dysmorphisms, brain and heart defects, hearing loss, hypotonia, as well as feeding problems. As new clinical symptom we add the West syndrome with hypsarrhythmia to the list of characteristic signs for ALG9-CDG. IEF of transferrin revealed a CDG-I pattern, indicating an early $\mathrm{N}$-glycosylation defect. $\mathrm{N}$-glycan analysis by MS further showed a reduction of several sugar moieties of serum glycoproteins in case of ALG9 deficiency. Furthermore, the level of de-fucosylated N-glycans was elevated in ALG9-CDG. Biochemical studies further revealed the distinctive accumulations of Man6GlcNAc2-PP-dolichol and Man8GIcNAc2-PP-dolichol in LLO analysis which could be assigned to the novel homozygous missense variant c.1460T>C (p.L487P) detected in both patients. Besides, we found the amount of ALG9 protein to be significantly reduced, whereas the mRNA level was significantly enhanced, which we rate as compensatory effect. Analysis of the protein level after cycloheximide treatment in patient and control fibroblasts revealed a faster degradation of ALG9 in patient cells due to variant p.L487P. We here also present the synopsis of clinical findings of our patients and of all other previously published ALG9CDG cases

Conclusion/Discussion: Our work adds new clinical, genetic and biochemical data and hereby helps to expand the knowledge about this rare $\mathrm{N}$-glycosylation disorder.

\section{P26}

Severity-adjusted evaluation of newborn screening on the metabolic disease course in cytosolic ureacycle disorders

Zielonka M. ', Kölker S. ', Scharre S. ', Seidl M. ', Gleich F.', Stützenberger N. ${ }^{1}$, Probst J.', Druck A. ', Nagamani S. ${ }^{2}$, Gropman A. ${ }^{3}$, Hoffmann G. ${ }^{\prime}$, Garbade S. ', Posset R. ${ }^{1}$

${ }^{1}$ Center for Pediatric and Adolescent Medicine, Division of Pediatric Neurology and Metabolic Medicine, University Hospital Heidelberg, Heidelberg, Germany; ${ }^{2}$ Department of Molecular and Human Genetics, Baylor College of Medicine and Texas Children's Hospital, Houston, Texas, USA; ${ }^{3}$ Children's National Health System and The George Washington School of Medicine, Washington, District of Columbia, USA,

Introduction: The implementation of newborn screening (NBS) programs for the cytosolic urea cylce disorders (UCDs) citrullinemia type 1 (CTLN1) and argininosuccinic aciduria (ASA) is subject to controversial debate. The aim of this study was to assess the impact of NBS on the metabolic disease course of affected individuals in a severity-adjusted manner.

Patient(s) and Methods: In 115 individuals with CTLN1 and ASA, we compared the severity of the initial hyperammonemic episode (HAE) and the frequency of (subsequent) HAEs with the mode of diagnosis. Based on a recently established functional disease prediction model, individuals were stratified according to their predicted severe or attenuated phenotype.

Results: NBS predominantly identified individuals with an attenuated phenotype of CTLN1 and ASA. Early identification by NBS enabled an efficient reduction of the severity of the initial HAE independent from the underlying phenotype, but was not associated with a reduced number of subsequent HAEs for both severe and attenuated phenotypes.

Conclusion/Discussion: The use of a functional disease prediction model enabled the severity-adjusted evaluation of NBS on the metabolic disease course in UCDs, which is of importance to avoid overestimation of the NBS effect. Future long-term studies will need to evaluate the clinical impact of this finding, especially with regard to mortality, as well as cognitive outcome and quality of life of survivors. We propose to use comparable severity-adjusted stratification methods to assess the impact of NBS for further inherited metabolic diseases in the future. 


\section{P27}

Cataracts and diarrhea of unknown cause: think of the treatable leukodystrophy Cerebrotendinous Xantomatosis (CTX)!

\author{
Oppermann I. ${ }^{1}$, Hempel M. ${ }^{2}$, Guder P. ${ }^{1}$, Santer R. ${ }^{1}$, Bley A. ${ }^{1}$ \\ 'University Children's Hospital, University Medical Center Hamburg-Eppendorf; ${ }^{2}$ Depart- \\ ment of Human Genetics, University Medical Center Hamburg-Eppendorf
}

Introduction: Cerebrotendinous Xantomatosis (CTX) is caused by an inherited deficiency of 27-sterol hydroxylase encoded by CYP27A1 leading to elevated cholestanol concentrations and variable clinical manifestations in patients. Many affected patients suffer from chronic diarrhea, cataracts and developmental abnormalities during childhood and adolescence and from a progressive leukodystrophy. Xanthomas and arteriosclerosis may appear in early adulthood. Treatment with chenodeoxycholic acid (CDCA) is an approved treatment for CTX in Germany.

Patient(s) and Methods: Analysis of four CTX cases concerning diagnostic results, symptoms and course of the disease.

Results: All four patients with genetically confirmed CTX showed elevated concentrations of cholestanol in blood and typical symptoms of CTX. Diagnosis was made between the age of 8 and 41 years. At the time of data analysis, one patient was 9 years old, three patients were between 30 and 47 years old. All four patients suffered from epileptic seizures with a spectrum from febrile seizures in childhood to a permanent epilepsy with need of antiepileptic treatment. Three out of four patients developed chronic diarrhea during childhood, three out of four showed developmental delay. Until adulthood all three adult patients developed bilateral cataracts and psychiatric symptoms. Only two out of three adult patients developed xanthomas. CMRIs of the adult patients showed pathognomonic white matter changes of the brain. One patients suffered from a cardiac infarction at the age of 39 years. All four patients started treatment with chenodeoxycholic acid supplementation. Diarrhea of two of these patients stopped immediately and also other clinical symptoms improved Conclusion/Discussion: CTX is a progressive leukodystrophy with multiple other organ manifestations if not treated early. Neonatal cholestasis, chronic diarrhea, cataracts and developmental abnormalities in childhood and adolescence can be early symptoms of a CTX.

It is conceivable that an early start of the approved oral chenodeoxycholic acid supplementation improves the course of the disease. Thus, early diagnosis by a newbornscreening that needs to be established will definitely be beneficial.

\section{P28}

\section{Simulation of glutaric acidemia type 1 in COS-7 cells- enzymatic activity and expression}

\author{
Yuan Y. ${ }^{1}$, Dimitrov B. ${ }^{1}$, Zielonka M. ${ }^{1}$, Kölker S. ${ }^{1}$ \\ ${ }^{1}$ Dietmar-Hopp Metabolic Center, Center for Child and Adolescent Medicine, Heidelberg \\ University Hospital
}

Introduction: Glutaric acidemia type 1 (GA1; OMIM, \#231670) is caused by deficiency of glutaryl-CoA dehydrogenase $(\mathrm{GCDH})$, an enzyme in the degradation pathway of lysine. Typical GA1 patients present among other things with striatal damage and dystonia. No evidence was found to support a correlation between residual enzyme activity and phenotype. This study aims to establish a feasible enzyme assay to facilitate diagnosis and to investigate phenotype prediction by exploring the correlation between the genotype, the remaining enzyme activity, biochemical specifications and potentially at least one of the patients' symptoms.

Patient(s) and Methods: The established GCDH activity assay utilizes ferricenium hexafluorophosphate as the electron acceptor and follows the reduction of ferricenium at $300 \mathrm{~nm}$. Mutated GCDH was transiently transfected into mammalian cells in accordance to 24 patient genotypes, including 6 homozygous (homozygous R128Q, P248L, E365K, R383C, R402 W and
A421V) and 3 compound heterozygous genotypes (R227P/R88C, R402 W/ A421V and R88C/A421V). Western blotting was performed to determine protein expression. The correlation between clinical data, protein expression and residual enzyme activity was analyzed.

Results: One heterozygous R227P/V400M (a known genotype with high residual activity), one heterozygous carrier of R128Q and one homozygous $\mathrm{R} 128 \mathrm{Q}$ showed $45 \%, 31 \%$ and $5 \%$ activity in comparison to the wild-type enzyme, respectively. In all the other simulated genotypes based on the patients' data, residual activities were below $5 \%$.

Based on protein expression relative to the expressed wild-type enzyme, genotypes could be categorized into 4 groups, $>90 \%$ (R383C/R383C, A421V/A421V, R88C/A421V), 50-90\% (P248L/P248L, R88C/R227P), 10$50 \%$ (E365K/E365K, R402W/A421V), <10\% (R128Q/R128Q, R402W/ $\mathrm{R} 402 \mathrm{~W})$. Patients in each group can present with either high- or low-excretors, acute- or insidious-onset, variable severity and onset time.

Conclusion/Discussion: The spectrophotometric assay of GCDH in the established mammalian expression system showed comparable results to GCDH assays performed in patient samples, thus providing a feasible approach for facilitating future diagnoses. GCDH expression among mutants did fluctuate widely. Further, no correlation between protein expression and the phenotypes was found, up to date.

\section{P29}

\section{The Austrian register of inherited metabolic disorders- An epidemiologic evaluation of the last 100 years}

\author{
Ramoser G. ${ }^{1}$, Caferri F. ${ }^{2}$, Radlinger B. ${ }^{3}$, Scholl-Bürgi S. ${ }^{1}$, Karall D. ${ }^{1}$, IMD A. ${ }^{6}$
}

'Medical University of Innsbruck, Clinic for Pediatrics I, Inherited Metabolic Disorders, Innsbruck, Austria; ' ${ }^{2}$ Medical University of Innsbruck, Clinic for Pediatrics III, Innsbruck, Austria; ${ }^{3}$ Medical University of Innsbruck, Clinic for Internal Medicine I, Innsbruck, Austria; ${ }^{6}$ Register Group

Introduction: Inherited metabolic disorders (IMDs) are a group of rare disorders with disruption of metabolic pathways. The Austrian register of inherited metabolic disorders started between 1985 and 1995. It aims to evaluate epidemiologic data and connect care providers and patients. Patient(s) and Methods: We included all patients with an IMD according to the SSIEM-classification. A minimal dataset consisting of birth date, sex, diagnosis, date of diagnosis, diagnose setting and clinical outcome was gathered. All Austrian departments of Pediatrics, Neurology and Internal Medicine were invited to register their patients. Reporting period ended with February 2021. As the first reported patient was born in 1921 observational data is available for up to 100 years.

Results: A total of 2631 patients were included. In February 2021, 1627 patients were still alive (61.8\%). Thus a minimal prevalence of 1.8 per 10,000 Austrian citizens was estimated for 2020 . While half of all currently alive patients were adults (median age 17.6 years), $86 \%$ of all patients received treatment at a Pediatrics department. $59.9 \%$ of all patients were diagnosed within the first year of life (median 1.92 months). There was a male predominance in our cohort ( $\mathrm{m}: \mathrm{f}$ ratio $=1.2: 1)$. Altogether 247 individual diagnoses were documented. The three most frequent diagnoses were Phenylketonuria (17\%), classical Galactosemia (6.6\%) and Biotinidase deficiency (4.2\%). Disorders of amino acid and peptide metabolism (31.1\%), disorders of energy metabolism (15.1\%) and lysosomal disorders (15.0\%) were the three most common SSIEM categories. Information about clinical outcome was available from $71.8 \%$ of all patients. $50.3 \%$ of patients lived without symptoms and in $35.3 \%$ of patients symptoms were reported. $13.9 \%$ of all patients have already died.

Conclusion/Discussion: Next to its epidemiologic value another aim of this register is an enhanced exchange between care providers and patients. With over 200 different individual diseases, we provide evidence for the heterogeneity of IMDs in Austria. As almost $50 \%$ of patients are adults, other medical disciplines than Departments of Pediadtrics need to be involved in patient care. 
P30

\section{Mechanistic explanation of the metabolite pattern in medium-chain acyl-CoA dehydrogenase deficiency (MCADD)-implications for newborn screening}

Fingerhut R. ${ }^{1}$, Schöberl D. ', Breuer S. ', Schultis W. ${ }^{1}$

'SYNLAB MVZ Weiden, Newborn Screening, Weiden, Germany,

Introduction: Abnormal findings, like dicarboxylic aciduria in MCADD, often led the way to a diagnosis and the respective explanation. However, the clear mechanistic explanation for certain findings are missing, or just not consolidated. For example why is hexanoyl glycine in urine the marker metabolite for MCADD, and not octanoyl glycine. The aim of this study was to merge the analytic findings with the enzymatic findings, to a complete mechanistic explanation of the metabolite pattern in MCADD. These results were used to search for the best metabolites and metabolite ratios for the detection of MCADD in dried blood spots (DBS).

Methods: The literature was searched for all diagnostic metabolites in urine, plasma, or DBS. In addition we searched for mechanistic explanations for the formation of the respective metabolites. With this information a list of diagnostic acylcarnitines and ratios of acylcarnitines was prepared. Then a scoring system was proposed and checked with 12,100 normal samples from newborns, 89 samples of 61 patients with confirmed MCADD deficiency, 488 false positive samples ( $\mathrm{fp}$ ) (based on octanoylcarnitine and the ratio $\mathrm{C} 8 / \mathrm{C} 12$ ), and 4018 samples of patients with PKU.

Results: The proposed scoring system contained the following metabolites and ratios: Hexanoyl- (C6), octanoyl- (C8), decanoyl- (C10), decenoylcarnitine (C10:1), the sum of those 4 medium-chain acylcarnitines (Sumof-MCAC), C8/C12, C8/C6, C8/C10, C8/C16, C8/C3DC, sum-of-MCAC/C12. Additional criteria were that $\mathrm{C} 8$ must be higher than $\mathrm{C} 10(\mathrm{C} 8>\mathrm{C} 10)$, and $\mathrm{C} 8$ must be higher than $\mathrm{C} 6(\mathrm{C} 8>\mathrm{C} 6)$. These analytes and ratios were ranked according to their proposed relevance, and the scoring points were assigned between +4 and -5 . The maximum was 21 scoring points, and the minimum -16. The MCADD cases all scored between 16-21, normal samples between 15-(-16), the fp scored between $15-(-10)$, and the treated PKU patients between 15-(-16).

Conclusion/Discussion: NBS for MCADD is widely incorporated into NBS programs worldwide. However, the utilised marker metabolites, and ratios are not unique and differ significantly between the programs. Also the positive predictive value varies significantly. The introduction of the described scoring system could reduce the recall rate to nearly $0 \%$, and raise the positive predictive value to $100 \%$.

\section{P31 \\ Flavin adenine dinucleotide (FAD)-dependent oxidoreductase domain-containing protein 1 (FOXRED1) deficiency: clinical spectrum and possible treatment options}

\section{Kock V. ${ }^{1}$, Haack T. ${ }^{2}$, Wittig I. ${ }^{3}$, Freisinger P. ${ }^{1}$}

'Department of Pediatrics and Adolescent Medicine, Kreiskliniken Reutlingen, Germany; ${ }^{2}$ Institute of Medical Genetics and Applied Genomics, University of Tübingen, Germany; ${ }^{3}$ Faculty of Medicine, Functional Proteomics, Goethe University Frankfurt, Germany

Introduction: FOXRED1 is a respiratory chain complex I specific assembly factor. The FAD-binding site is involved in electron transfer. Mutations in FOXRED1 lead to partial loss of function and cause defects in complex I biogenesis resulting in mitochondrial dysfunction. The clinical picture is variable but mostly associated with Leigh-like disease. To date 6 patients have been described. No proven therapy is available so far.

Patient(s) and Methods: Patient A, first child of consanguineous parents, was a premature and small for gestational age. He had a severe course with developmental delay, seizures, pulmonary arterial hypertension and lactic acidosis in the first year of life. A homozygous missense variant in the FOXRED1 gene was identified by whole exome sequencing (WES). He died at the age of 21 months.

Patient $B$ and $C$ are siblings and of irakian origin. Because of isolated mental retardation WES was performed. A homozygous mutation in FOXRED1 was found. To confirm the diagnosis in an otherwise mild phenotype, a muscle biopsy was performed which showed a clearly reduced complex 1 activity. The brothers are adults and have still a similar, mild clinical course. Riboflavin is precursor for flavin adenine dinucleotide. Beneficial effects of riboflavin treatment were already shown in acyl-CoA dehydrogenase family member 9 (ACAD9) patients. ACAD9 is also a FAD-dependent complex I assembly factor. In vitro treatment of fibroblasts from ACAD9 patients with riboflavin shows improved assembly of complex 1 subcomplexes. All our patients were treated with riboflavin $(10 \mathrm{mg} / \mathrm{kg} / \mathrm{d})$. The effect will now be tested in vitro on fibroblasts from FOXRED1 patients.

Results: Conclusion/Discussion: As FOXRED1 is a flavin-dependent assembly factor for complex 1 formation, treatment with riboflavin could have also beneficial effect in FOXRED1 patients.

\section{P32}

Pyruvate dehydrogenase E3 binding protein deficiency: positive outcome in a preterm baby treated with ketogenic diet

\section{Kock V. ${ }^{1}$, Trips T. ${ }^{2}$, Fischer A. ', Haack T. ${ }^{3}$, Freisinger P. ${ }^{1}$}

'Department of Pediatrics and Adolescent Medicine, Kreiskliniken Reutlingen, Germany; ${ }^{2}$ Department of Pediatrics and Adolescent Medicine, Klinikum Traunstein/Bezirkskrankenhaus Kufstein, Germany/Austria; ${ }^{3}$ nstitute of Medical Genetics and Applied Genomics, University of Tübingen, Germany

Introduction: Pyruvate dehydrogenase (PDH) E3 binding protein defect (OMIM 245349) is a rare cause of PDH-complex deficiency and is due to biallelic pathogenic variants in PDHX. Patients usually present with neonatal lactic acidosis, muscular hypotonia, global developmental delay, spasticity and epilepsy.

Patient(s) and Methods: Our patient, a girl, was delivered after 30 weeks of gestation by caesarian section due to partial placental abruption. The initial lactic acidosis was attributed to perinatal distress but persisted during the following period. At day 14, she suffered from intracranial hemorrhage grade 2 . A comprehensive metabolic analysis showed elevated alanine but was inconclusive. Suspecting a mitochondrial disorder the nutritional lipid content was increased to $55 \%$ of kilocalories (kcal).

Results: Urgent whole exome sequencing (WES) revealed a known pathogenic homozygous variant in PDHX (R446X) already described in Roma families. On day 35 the fat content in the patient's nutrition was progressively increased to $80 \%$ (of $\mathrm{kcal}$ ) respecting the requirements of prematurity. This treatment was well tolerated and lactate levels decreased (2-4 mmol/l). MRI of the brain at 52 days of age showed no structural abnormalities and delayed myelination adequate for her age. Ketogenic diet was continued. At age of two years, she showed a discrete developmental delay. Head circumference was at the third percentile and brain MRI was normal.

Conclusion/Discussion: Patients with the Roma founder mutation in PDHX (R446X, homozygous) are well characterized and usually suffer from severe developmental delay, dystonia and epilepsy after 1 year of age. Our patient, who in addition was a 30 weeks premature, presented only a mild developmental delay at age two years. We assume that this is the result of very early initiation of ketogenic diet. This example illustrates that ketogenic diet is even possible in preterm babies if there is an urgent indication. Furthermore, it shows that early and urgent WES can be extremely helpful for therapeutic decisions. 


\section{P33}

\section{Secondary glutathione deficiency in hyperinflammatory syndrome}

Zühlsdorf A. ${ }^{1}$, Masjosthuesmann K. ${ }^{1}$, Rödiger M. ${ }^{1}$, Marquardt T. ${ }^{1}$

${ }^{1}$ Klinik für Kinder- und Jugendmedizin, Allgemeine Pädiatrie, Universitätsklinikum Münster

Introduction: 5-Oxoprolinuria occurs during glutathione deficiency and can be caused by several factors such as metabolic disorders, other illnesses and different medications. Glutathione has a key role in our metabolism as antioxidant and is essential in many metabolic pathways. With continuous oxidative stress e.g. during sepsis glutathione levels are rapidly reduced. Glutathione deficiency may lead to cell damage caused by oxidative stress resulting in metabolic acidosis, hemolytic anemia and neurological symptoms.

Patient(s) and Methods: Our patient was admitted to pediatric intensive care unit (PICU) due to suspicion of febrile infection related refractory epilepsy syndrome (FIRES) or multisystem inflammatory syndrome in children (MIS-c) presenting with hyperinflammation and enterocolitis, in course suffering from several sepsis-like episodes. After several weeks of PICU treatment with already improving general conditions she presented with a worsening neurological status including complex focal seizures. Results: Her overall condition was most likely caused by a MIS-c associated after COVID19. Complex work up revealed extraordinarily high levels of 5-Oxoproline in her urine, genetic work up did not reveal any mutations affecting glutathione metabolism. Treatment with high dosage vitamin $\mathrm{E}$ and $C$ was initiated, her neurological status improved, urine levels of 5-Oxoproline normalized and substitution was discontinued. 5-Oxoproline remained normal in due course. Up until today she suffers from occasional seizures.

Conclusion/Discussion: Secondary glutathione deficiency may occur in critically ill patients requiring extensive PICU treatment and should be considered in ICU patients with metabolic acidosis, hemolysis or neurological worsening with unknown cause. Patients can be treated with substitution of vitamin $\mathrm{E}$ and $\mathrm{C}$ as antioxidative medication, which may protect them from long term effects due to diminished protection against oxidative stress initiated by extensive medication as well as disease patterns requiring intensive care treatment.

\section{P34}

\section{A case of glutaric aciduria type III (GA-III)}

Woidy M. ${ }^{1}$, Tsiakas K. ${ }^{1}$, Murko S. ${ }^{2}$, Santer R. ${ }^{1}$

${ }^{1}$ Children's Hospital, University Medical Center Hamburg, Germany; ${ }^{2}$ Neonat Screen Metab Diagn Unit, University Medical Center Hamburg, Germany

Introduction: Elevated urinary excretion of glutaric acid can be found in genetic defects of glutaryl-CoA dehydrogenase $(G C D H, G A-I)$ or its cofactor system (ETFA, ETFB, ETFDH; GA-II). Here we describe the incidental finding of glutaric aciduria due to impaired mitochondrial CoA transfer from succinyl CoA to glutarate, GA-III.

Patient(s) and Methods: Case study: of a 3-yr-old girl detected in neonatal screening for biotinidase deficiency and the incidental finding of glutaric acid in urine.

Results: Following neonatal screening biotinidase deficiency was subsequently confirmed by enzymatic analyses (residual activity 2-8\%) and genetic testing (BTD [p.L215F];[p.A271S]). Neither lactic acidosis nor organic aciduria by typical acids were detected before or after biotin treatment. However, small amounts of glutaric acid (56-72 $\mu \mathrm{mol} / \mathrm{mmol}$ Krea, no 3$\mathrm{OH}$-glutaric acid) were consistently found in urine. Neonatal screening for and repeated analysis of acylcarnitines was unremarkable including CO and C5DC. Targeted genetic testing detected compound heterozygosity for two variants within the succinyl-CoA-glutarate-transferase gene (SUGCT [p.S246P];[p.R336 W]).
Conclusion/Discussion: GA-III has long been considered a non-disease and also our patient has remained asymptomatic. Only recently, however, it has been claimed from experiments in mice that SUGCT knock-out is associated by changes in the gut microbiome responsible for an obesityrelated phenotype accompanied by lipid accumulation in kidney and liver.

\section{P35}

Case report: Use of glycomacropeptide as a single protein supplement in a pregnancy of a patient with phenylketonuria

\author{
Lier D. ${ }^{1}$, Kock V. ${ }^{1}$, Wallner S. ${ }^{2}$, Freisinger P. ${ }^{1}$
}

'Department of Pediatrics and Adolescent Medicine, Kreiskliniken Reutlingen, Germany; ${ }^{2}$ MVZ Laborärzte Singen, Germany

Introduction: In women with Phenylketonuria (PKU) blood phenylalanine (Phe) levels should be within a target range of $120-360 \mu \mathrm{mol} / \mathrm{l}$ during pregnancy to prevent maternal PKU syndrome in the child. This requires a strict low Phe dietary treatment, which consists of natural low protein products, special low Phe products and a PKU protein supplement (PS) based on aminoacids or glycomarcropeptide (GMP). Some patients prefer GMP e. g. due to its palatability and digestibility.

We report a first pregnancy in a woman with classical PKU exclusively using GMP as PS.

Patient(s) and Methods: Report of metabolic control as well as dietary intake in a planned pregnancy of a classical PKU patient.

Results: Prior to the current pregnancy, the patient's mean blood Phe concentrations was $428 \mu \mathrm{mol} / \mathrm{I}$ (SD: 139) (20 tests in 25 months). Blood Phe concentrations $<360 \mu \mathrm{mol} / \mathrm{l}$ could be reached with a Phe intake of about $620 \mathrm{mg} / \mathrm{d}$. The low Phe diet was supplemented with $80 \mathrm{~g}$ of protein equivalents from GMP, which was fully included into the daily Phe tolerance. During the first trimester the patient had mean blood concentrations of $311 \mu \mathrm{mol} / \mathrm{l}$ Phe (SD: 121) and $42.8 \mu \mathrm{mol} / /$ Tyrosine (Tyr) (SD: 8) (23 tests). In the 4th week and between 10th and 12th week of gestation the patient had two episodes of elevated blood Phe levels. The highest blood Phe concentration was $516 \mu \mathrm{mol} / \mathrm{l}$. Due to increased blood Phe the Phe intake was reduced to a minimum of $212 \mathrm{mg} / \mathrm{d}$.

In in the second trimester mean blood concentrations of $194 \mu \mathrm{mol} / \mathrm{I}$ Phe (SD: 69$)$ and $42.5 \mu \mathrm{mol} / \mathrm{l}$ Tyr (SD: 10) were measured (20 samples). In the third trimester $184 \mu \mathrm{mol} / \mathrm{l}$ Phe (SD: 59 ) and $45.3 \mu \mathrm{mol} / \mathrm{l} \mathrm{Tyr} \mathrm{(SD:} 25$ ) (14 samples), respectively. Phe intake was increased up to $1900 \mathrm{mg} / \mathrm{d}$ throughout the pregnancy.

Conclusion/Discussion: Our patient managed her pregnancy on GMP as exclusive PS without complications. The main challenge was that the Phe intake had to be reduced due to elevated blood levels. However, as long as the Phe content of GMP is included into the daily tolerance, the use of GMP as a single PS during pregnancy is possible.

\section{P36}

Dietary therapy of PKU: Sirtuin-activity in glycomacropeptide-based products and classical amino acid mixtures

Stahmer L. ${ }^{1}$, Hase K. ${ }^{1}$, Shammas H. ${ }^{1}$, Meyer U. ${ }^{1}$, Das A. ${ }^{1}$

'Medizinische Hochschule Hannover

Introduction: Glycomacropeptide-based products (GMP) are used as an alternative to classical amino acid mixtures (AAM) as protein supplements in the dietary treatment of PKU-patients. GMP are more palatable and cause less intestinal problems than AAM. Furthermore, GMP is supposed to result in enhanced, sustained satiety. Recently, we have found sirtuin activities (SIRT 1 and SIRT 3) in human breast milk which is regarded as the 'gold-standard'for feeding neonates and infants. Sirtuins are known to impact intestinal metabolism as well as the gut-microbiome and metabo- 
lome. Finally, sirtuins could be taken up by the blood circulation and regulate metabolism, cell proliferation and inflammation as well as longevity. We hypothesized that sirtuin activities differ in GMP and AAM.

Patient(s) and Methods: An aqueous phase and a cell pellet were obtained by centrifugation of GMP and AAM, respectively. Enzyme activities of SIRT 1 and SIRT 3 were assayed by fluorometry. Activities in the different products were standardized by calculating the ratio of enzyme activity and protein content in the ready to drink GMP or AAM.

Results: In the ready to drink product, GMP contained higher specific SIRT 1- and SIRT 3-activities compared to AAM. SIRT 1-activity in GMP was 180 $\mathrm{U} / \mathrm{g}$ in the aqueous phase and $410 \mathrm{U} / \mathrm{g}$ in the pellet while activity in AAM was $20 \%$ of the activity in GMP. For SIRT3, enzyme activity in GMP was $1,800 \mathrm{U} / \mathrm{g}$ in the aqueous phase and 3,900 $\mathrm{U} / \mathrm{g}$ in the pellet, in AAM activities were $1,600 \mathrm{U} / \mathrm{g}$ in the aqueous phase and $1,100 \mathrm{U} / \mathrm{g}$ in the pellet. There were considerable differences of sirtuin activities between different AAM-products.

Conclusion/Discussion: GMP show higher specific enzyme activities for SIRT 1 and SIRT 3, both in the aqueous phase and the cell pellet compared to AAM. The pathophysiological consequences of these findings are still unclear. Sirtuins may impact the gut microbiome and metabolome or have an effect on the metabolism of intestinal cells, alternatively, sirtuins may be taken up by blood and impact the function of different organs. Further experimentation is required to elucidate the function of sirtuins in the nutrition of neonates and infants.

\section{P37}

\section{In frame biallelic GCSH duplication in a patient with an attenuated nonketotic hyperglycinemia}

Tsiakas K. ${ }^{1}$, Murko S. ${ }^{2}$, Hempel M. ${ }^{3}$, Mayr J. ${ }^{1}$, Rodriguez Pombo P. ${ }^{4}$, Van Hove J. ${ }^{5}$, Santer R.

'Dept Pediatr, Univ Med Ctr Eppendorf, Hamburg, Germany; ${ }^{2}$ Metab Lab, Univ Med Ctr Eppendorf, Hamburg, Germany; ${ }^{3}$ nst Hum Gen, Univ Med Ctr Eppendorf, Hamburg, Germany; ${ }^{4}$ Diagn Ctr Mol Dis, Universidad Autónoma Madrid, Spain; ${ }^{5}$ Children's Hospital University of Colorado, Aurora, USA

Introduction: $\mathrm{GCSH}$, encoding the $\mathrm{H}$ protein of the glycine cleavage complex, is also involved in lipoate synthesis. GCSH variants have been proposed in the past as a possible cause of nonketotic hyperglycinemia (NKH). So far, there is only one very recent publication describing three individuals with a single biallelic genetic variant in GCSH associated with a well characterized clinical, biochemical and radiological picture of a variant form of NKH (Majethia et al. 2021, PMID 33890291). Here, we report another patient with an attenuated NKH phenotype and a novel pathogenic GCSH variant.

Patient(s) and Methods: Case Study of a 19-month-old male who presented in the 2 nd week of life with apnoic episodes. Common differential diagnoses were excluded. Glycine concentrations in plasma und CSF were elevated. Newborn brain MRI showed restricted diffusion of bilateral motor corticospinal tracts. The patient recovered rapidly under treatment with sodium benzoate and dextromethorphan and remained stable. Currently, he suffers from a dystonic movement disorder and moderate global developmental delay without feeding problems and epilepsy so far. Glycine concentrations in CSF and plasma at the age of 15 months have remained elevated. Homozygosity for an in frame duplication, encompassing the last two exons 4 und 5 of GCSH, was shown by NKH panel analysis. Trio whole exome sequencing confirmed the above finding und excluded pathogenic variants in other genes. Functional experiments for the evidence of pathogenicity of the observed variant have not been finalized yet.

Conclusion: With this report, we propose that GSCH deficiency causes an attenuated and altered NKH phenotype. Thus, we extend the genetic and phenotypic spectrum of this metabolic disorder.
P38

\section{Metabolic decompensation of adult IEM patients by COVID-19 vaccination: a case series}

Köhler J.', Schöler D.', Afzal S. ', Förner B.', May P.', Keitel-Anselmino V.', Lüdde T.', vom Dahl S. ${ }^{1}$

'Klinik für Gastroenterologie, Hepatologie und Infektiologie, Universitätsklinikum, Düsseldorf

Introduction: The current COVID-19 pandemic is particularly challenging for patients with inborn errors of metabolism. Experience with the protective vaccination and its effect on the metabolism of patients with IEM is not widely accessible. Although experience with protective vaccination in IEM patients is generally good, two cases of patients with aminoacidopathias and metabolic derailment triggered by COVID vaccination are described.

Patient(s) and Methods: Two cases of patients with aminoacidopathias and metabolic derailment triggered by COVID vaccination are described.

Results: Patients: Patient \#1 was an 18 year old female diagnosed with citrullinemia during early infancy and had shown good metabolic control during childhood and early adulthood. Hyperammonic metabolic derailments had not been present during the last years. The night after vaccination with BNT162b2 (Comirnaty ${ }^{\circledast}$, Biontech/Pfizer), the patient developed fever, vomiting and diarrhoea and presented immediately to our ER with gradually deteriorating somnolence. Blood ammonia was elevated to $149 \mu \mathrm{g} / \mathrm{dl}$. The patient was admitted to the ICU for monitoring. There was no indication of systemic infection. Stabilisation was achieved by parenteral nutrition to prevent catabolism, fluid administration and protein restriction. The patient had to stay in hospital for 1 week and has almost recovered.

Patient \#2 was a 29 year old female with HMG-CoA-lyase deficiency diagnosed at age 3 who had shown several hypoglycemic, non-ketotic hyperlactataemic episodes during infancy and early adulthood, but metabolically stable for 2 years before current admission. As an additional risk factor the patient was underweight with a BMI of $17.6 \mathrm{~kg} / \mathrm{m}^{2}$. The patient received a vaccination with BNT162b2 (Comirnaty ${ }^{\circledast}$, Biontech/Pfizer) two days before her external emergency hospital admission. Following initial discomfort with a phase of non-hyperammonemic hyperlactataemia, rhabdomyolysis, tachypnoea with increasing hepatomegaly and a subacute hepatitis developed and the patient was transferred to our institution. Currently, the patient was non-confuse, her blood lactate was $18 \mathrm{mmol} / \mathrm{l}$, GPTmax was $221 \mathrm{U} / \mathrm{l}$, and CK increased to $18895 \mathrm{U} / \mathrm{l}$, with sinus tachycardia of $145 / \mathrm{min}$ and respiratory frequency of $36 / \mathrm{min}$. Full intensive care therapy with infusion therapy, buffering, iv carnitine supplementation and prophylactic antibiotic therapy is ongoing.

Conclusion/Discussion: Both cases impressively demonstrate the potential danger of COVID-19 vaccination for patients with IEM. Patients should be informed of the potential risk of metabolic derailment prior to vaccination and should be carefully advised and monitored. 